[white paper]

Diamond Open Access

\title{
Personal Handbook of Logic
}

\author{
Open Mathematics Collaboration*†
}

February 9, 2023

\begin{abstract}
This is a personal collection of definitions and results from first-order logic.
\end{abstract}

keywords: personal handbook, first-order logic

The most updated version of this white paper is available at https://osf.io/8wck9/download https://zenodo.org/record/5594984

\section{Preamble}

1. Mathematics is the Queen of the Sciences (Gauss).

2. Logic is the Queen of Mathematics.

*All authors with their affiliations appear at the end of this white paper.

${ }^{\dagger}$ Corresponding author: mplobo@uft.edu.br|Open Mathematics Collaboration 


\section{Contents}

Metalinguistic Symbols

Symbols and Syntax

List of Symbols

First-order Language

Terms

Formulas

Atomic Formulas

Complexity

Mathematical Induction

Free Variables

Sentences

Structures

Variable Assignment Function

Term Assignment Function

Satisfaction

True Sentences

On the equality of term assignment functions

Satisfaction of a formula with different variable assignment functions 
Satisfaction for all variable assignment functions

Model (formula)

True Sentences

Satisfaction of formulas with the connective "and"

Satisfaction with the existential quantifier

Substitution into a Term

Substitution into a Formula

A term substitutable for a variable in a formula

Logical Implication (sets of formulas)

Valid Formulas

Universal Closure of a Formula

On the validity of a conditional statement of formulas

"Bottom-up" Deduction

"Top-down" Deduction

Decidable Set of Axioms

(Non)Logical Axioms

Equality Axioms

Quantifier Axioms

Rules of Inference 
Propositional Consequence: Definition

Propositional Consequence: Tautology

Propositional Consequence: Extension to First-order Logic

Rule of Inference of type (PC)

Rules of Inference of type (QR)

On the validity and tautology of formulas

List of requirements for axioms and rules of inference

Logical Axioms: Valid

Rule of Inference: Theorem

Soundness Theorem

When a variable is not free in a formula

Variable Assignment Functions and Substitutions

Term substitution in the $x$-modification of the assignment function

Equality: Equivalence Relation

A set of formulas proves a formula if and only if it proves the formula for all variables

Adding/deleting a universal quantifier

The Deduction Theorem

Proofs by Contradiction 
Unary Relation Symbol

Binary Relation Symbol

Two unary relation symbols

Complete Deductive System

(In)Consistent

Completeness Theorem

Soundness + Completeness

Compactness Theorem

(Finitely) Satisfiable

Finite subset of a set of formulas

First-order Sentences: Natural Numbers

Theory of a Structure

Substructure

Elementary Substructure/Extension

Truth in elementary substructure/extension

Condition for an elementary substructure

Hilbert Axiomatic System

Axioms of the Hilbert-style Calculus

Inference Rule of the Hilbert-style Calculus 
Sequent Systems: Classical Logic

Proofs and Provability (in LK)

Rules for single formulas (in LK)

Multisets of Formulas

Logical constant 0

Orthologic

Intuitionistic Reasoning

Intuitionistic Propositional Logic: Syntax

Axiom Schema for Intuitionistic Propositional Logic

Modal operators

Decision problem

Undecidable

Word problem

Natural Deduction in Heyting Semantics

Lambda Calculus: Types

Lambda Calculus: Terms

Lambda Calculus: Denotational significance

System of equations in lambda calculus:

Consistent and decidable

Conversion 
The Curry-Howard Isomorphism

The Normalization Theorem

The lambda-calculus: Introduction

The lambda-calculus: Formal system

The lambda-calculus: Informal interpretation

Lambda-terms: Length, occurrence, scope, free and bound variables, substitution

Lambda-terms: Change of bound variables, congruence

Lambda-terms: Simultaneous substitution

Lambda-terms: $\beta$-reduction

Lambda-terms: $\beta$-normal form

Lambda-terms: $\beta$-equality

Simple typing, Church-style

Typed $\lambda$-calculus

The Sequent Calculus LJ

The Sequent Calculus LJT

Translation of proofs from LJ to LJT

Proofs in Natural Deduction

Proof of sequents in LK

Proof of sequents in LJ and in LJT 
Quantum Logics: Introduction 


\section{Introduction}

3. This handbook is mostly intended for consultation.

4. Each section can be read independently.

5. Due to (3) and (4), there are redundancies in many of the definitions.

6. At the beginning of each section, we present the references used.

\section{Metalinguistic Symbols}

7. $[1,2]$

8. A metalinguistic symbol is not part of the language.

9. The symbol $:=$ means that what is on the left side is defined by the right side of it.

10. The symbol : E means that the strings of symbols (within a language) on each side of it are identical.

11. $\vdash$ means deduction, logically implies

12. $\models$ means satisfy, truth (if there is a structure on the left), logical implication (if there is a set of sentences on the left), model

13. $\perp($ read false or eet $):=$ contradictory sentence

14. The symbol $\sim \succ$ appears for pedagogical purpose for the sake of abbreviating an explanation; it can be read as from, of, with, leads to, in which, etc.

\section{Symbols and Syntax}

15. $[1,2]$ 
16. syntax $:=$ symbols (of a language)

17. string $:=$ string of symbols $:=$ a sequence of symbols

\section{List of Symbols}

18. $[1,2]$

19. $\in:=$ membership relation

$20 . \notin:=$ negation of the membership relation

21. $\underline{\vee}:=$ exclusive or

22. $\subseteq:=$ subset, substructure

23. $\prec:=$ elementary substructure/extension

24. $\cup:=$ union of sets

25. $\cap:=$ intersection of sets

26. $\emptyset:=$ empty set

27. $f \uparrow_{A}:=$ restriction of the function $f$ to the domain $A$

\section{First-order Language}

28. $[1,2]$

29. first-order language $:=$ infinite collection of distinct symbols (no one of which is properly contained in another) separated into the following:

(a) Parentheses: (,)

(b) Connectives: $\vee, \neg$ 

(c) Quantifier: $\forall$
(d) Variables (one for each positive integer $n$ ): $v_{1}, v_{2}, \ldots, v_{n}, \ldots$ Vars $=\left\{v_{1}, v_{2}, v_{3}, \ldots\right\}:=$ set of variable symbols
(e) Equality: $=$
(f) Constant: Some set of zero or more symbols
(g) Function: For each positive integer $n$, some set of zero or more $n$-ary function symbols
(h) Relation: For each positive integer $n$, some set of zero or more $n$-ary relation symbols

\section{Terms}

30. $[1,2]$

31. term of $\mathcal{L}:=$ nonempty finite string $t$ of symbols from $\mathcal{L}$ such that either:

(a) $t:=$ constant symbol $(c)$, or

(b) $t:=$ variable $(v)$, or

(c) $t: \equiv f t_{1} t_{2} \ldots t_{n}$, where

$f:=n$-ary function symbol of $\mathcal{L}$ and

$t_{i}:=$ term of $\mathcal{L}$.

32.

$$
t:=c \underline{\vee} v \underline{\vee} f
$$

33. $\mathcal{L}:=$ first-order language

34. $\mathcal{L}$-symbols $:=$ symbols of a language $\mathcal{L}$

35. Note that (31.c) is a definition by recursion, since $t$ is a term if it contains substrings that are terms.

36. substring $:=$ subset of a string 


\section{Formulas}

37. $[1-3]$

38. formulas := assertions about the objects of the structure (model)

39. formula of $\mathcal{L}: \equiv$ nonempty finite string $\phi$ of symbols from $\mathcal{L}$ such that either:
(a) $\phi: \equiv=t_{1} t_{2}$, or
(b) $\phi: \equiv R t_{1} t_{2} \ldots t_{n}$, or
(c) $\phi: \equiv(\neg \alpha)$, or
(d) $\phi: \equiv(\alpha \vee \beta)$, or
(e) $\phi: \equiv(\forall v)(\alpha)$.

40. $\mathcal{L}:=$ first-order language

41. $t_{1}, t_{2}, \ldots, t_{n}:=$ terms of $\mathcal{L}$

42. $R:=n$-ary relation symbol of $\mathcal{L}$

43. $\alpha, \beta:=$ formulas of $\mathcal{L}$

44. $v:=$ variable

45. Note that $(39 . c, d, e)$ are definitions by recursion, since $\phi$ is a formula if it contains other formulas.

46. In (39.e), we say that the scope of the quantifier $\forall$ is $\alpha$.

47. $p \wedge \neg p$ has two formula occurrences of $p$

\section{Atomic Formulas}

48. $[1,2]$ 
49. atomic formula of $\mathcal{L}:=$ nonempty finite string $\phi$ of symbols from $\mathcal{L}$ such that either:

$$
\begin{aligned}
\text { (a) } \phi: \equiv=t_{1} t_{2}, \text { or } \\
\text { (b) } \phi: \equiv R t_{1} t_{2} \ldots t_{n} .
\end{aligned}
$$

50. $\mathcal{L}:=$ first-order language

51. $t_{1}, t_{2}, \ldots, t_{n}:=$ terms of $\mathcal{L}$

52. $R:=n$-ary relation symbol of $\mathcal{L}$

53. Atomic formulas are the primitives (not defined under recursion).

54. atom $:=$ atomic formula

55. literal $:=$ atom or its negation

\section{Complexity}

56. $[1,2]$

57. simpler formula $:=$ fewer number of connectives/quantifiers

58. simpler formula := subformula of a more complex formula

\section{Mathematical Induction}

59. $[1,2]$

60. proof by induction on the structure (complexity) of the formula

\section{Free Variables}

61. $[1,2]$ 
62. $v:=$ free in $\phi$ if

(a) $\phi$ is atomic and $v$ occurs in (is a symbol in) $\phi$, or

(b) $\phi: \equiv(\neg \alpha)$ and $v$ is free in $\alpha$, or

(c) $\phi: \equiv(\alpha \vee \beta)$ and $v$ is free in at least $\alpha$ or $\beta$, or

$(d) \phi: \equiv(\forall u)(\alpha)$ and $v$ is not $u$ and $v$ is free in $\alpha$.

63. $u, v:=$ variables

64. $\phi, \alpha, \beta:=$ formulas

\section{Sentences}

65. $[1,2]$

66. sentences $:=$ formulas that can be either true or false (with no free variables)

67. There are no free variables in the definition of a sentence so that it can be either true or false.

68. $\mathcal{L}:=$ first-order language

\section{Structures}

69. $[1,2]$

70. $\mathfrak{A}:=\operatorname{set} A$ together with

(a) an element $c^{\mathfrak{A}}$ of $A$, for each constant symbol $c$ of $\mathcal{L}$,

(b) a function $f^{\mathfrak{A}}: A^{n} \rightarrow A$, for each $n$-ary function $f$ of $\mathcal{L}$, and

(c) an $n$-ary relation $R^{\mathfrak{A}}$ on $A$ (i.e., a subset of $A^{n}$ ), for each $n$-ary relation symbol $R$ of $\mathcal{L}$. 
71.

$$
\mathfrak{A}=\left(A, c^{\mathfrak{A}}, f^{\mathfrak{A}}, R^{\mathfrak{A}}\right)
$$

72. $c^{\mathfrak{A}} \in A, \quad f^{\mathfrak{A}}: A^{n} \rightarrow A, \quad R^{\mathfrak{A}} \subseteq A^{n} ; \quad A \neq \emptyset$

73. $\mathcal{L}:=$ first-order language

74. $\mathfrak{A}:=\mathcal{L}$-structure

75. $A:=$ the universe of $\mathfrak{A}$

76. Note that the variables are not part of the definition (70).

\section{Variable Assignment Function}

77. $[1,2]$

78. assignment functions

(i) begin the process of tying together the symbols with the structures)

(ii) formalize the interpretation of a term/formula in a structure

79. variable assignment function into $\mathfrak{A}:=$ function $s$ that assigns to each variable an element of $A$,

$$
s: \text { Vars } \rightarrow A
$$

80. $\operatorname{Vars}:=$ set of variable symbols (domain)

81. $A:=$ universe of $\mathfrak{A}$ (codomain)

82. $s[x \mid a](v)= \begin{cases}s(v), & \text { if } v \text { is a variable other than } x \\ a, & \text { if } v \text { is the variable } x\end{cases}$

83. $s:=$ variable assignment function into $\mathfrak{A}$ 
84. $x:=$ variable; $a \in A$

85. $s[x \mid a](v):=x$-modification of the assignment function $s$ 86. In $s[x \mid a](v), x$ is assigned to $a$.

87. $\mathcal{L}:=$ first-order language

88. $\mathfrak{A}:=\mathcal{L}$-structure

\section{Term Assignment Function}

89. $[1,2]$

90. $\bar{s}:=$ term assignment function generated by $s$
(a) $(t:=$ variable $) \rightarrow(\bar{s}(t)=s(t))$
(b) $(t:=$ constant symbol $c) \rightarrow\left(\bar{s}(t)=c^{\mathfrak{A}}\right)$
(c) $\left(t:=f t_{1} t_{2} \ldots t_{n}\right) \rightarrow\left(\bar{s}(t)=f^{\mathfrak{A}}\left(\bar{s}\left(t_{1}\right), \bar{s}\left(t_{2}\right), \ldots, \bar{s}\left(t_{n}\right)\right)\right)$

91. $\bar{s}$ (term) is the generalization of $s$ (variable).

92. Note that $\bar{s}$ is defined recursively.

93. set of $\mathcal{L}$-terms $:=$ domain of $\bar{s}$

94. $A:=$ codomain of $\bar{s}$

95. $s:=$ variable assignment function into $\mathfrak{A}$

96. $c^{\mathfrak{A}} \in A$

97. $\mathcal{L}:=$ first-order language

98. $\mathfrak{A}:=\mathcal{L}$-structure 


\section{Satisfaction}

99. $[1,2]$

100. satisfaction $:=$ truth

101.

$(\mathfrak{A} \models \phi[s]):=\mathfrak{A}$ satisfies $\phi$ with assignment $s$ if

(i) $\left(\phi: \equiv=t_{1} t_{2}\right) \wedge\left(\bar{s}\left(t_{1}\right)\right.$ is the same element of $A$ as $\left.\bar{s}\left(t_{2}\right)\right)$, or

(ii) $\left(\phi: \equiv R t_{1} t_{2} \ldots t_{n}\right) \wedge\left(\left(\bar{s}\left(t_{1}\right), \bar{s}\left(t_{2}\right), \ldots, \bar{s}\left(t_{n}\right)\right) \in R^{\mathfrak{A}}\right)$, or

(iii) $(\phi: \equiv \neg \alpha) \wedge(\mathfrak{A} \not \models \alpha[s])$, or

(iv) $(\phi: \equiv \alpha \vee \beta) \wedge((\mathfrak{A} \models \alpha[s]) \vee(\mathfrak{B} \models \beta[s]))$, or

$(v)(\phi: \equiv \forall x \alpha) \wedge(\forall a \in A: \mathfrak{A} \models \alpha[s(x \mid a)])$.

102. $\mathcal{L}:=$ first-order language

103. $\mathfrak{A}:=\mathcal{L}$-structure

104. $\phi:=\mathcal{L}$-formula

105. $s:$ Vars $\rightarrow A$

106. $s:=$ variable assignment function into $\mathfrak{A}$

107. Vars $:=$ set of variable symbols

108. $A:=$ universe of $\mathfrak{A}$

109. $(\mathfrak{A} \models \Gamma[s]) \equiv(\forall \gamma \in \Gamma: \mathfrak{A} \models \gamma[s])$

110. $\Gamma:=$ set of $\mathcal{L}$-formulas 


\section{True Sentences}

111. $[1,2]$

112.

$$
(\sigma \text { is true in } \mathfrak{A}) \leftrightarrow(\mathfrak{A} \models \sigma[s])
$$

113. $\sigma:=$ sentence

114. $\mathfrak{A}:=$ structure

115. $s:=$ variable assignment function into $\mathfrak{A}$

116. Note that the definition of satisfaction is relative to an assignment function.

\section{On the equality of term assignment functions}

117. $[1,2]$

118.

$$
\left(\forall v \in t: s_{1}(v)=s_{2}(v)\right) \rightarrow\left(\overline{s_{1}}(t)=\overline{s_{2}}(t)\right)
$$

119. $\mathfrak{A}:=$ structure

120. $v:=$ variable

121. $s_{1}, s_{2}:=$ variable assignment functions into $\mathfrak{A}$

122. $t:=$ term

Satisfaction of a formula with different variable assignment functions

123. $[1,2]$ 
124.

$$
\left(\forall v \in \phi: s_{1}(v)=s_{2}(v)\right) \rightarrow\left(\mathfrak{A} \models \phi\left[s_{1}\right] \leftrightarrow \mathfrak{A} \models \phi\left[s_{2}\right]\right)
$$

125. $\mathfrak{A}:=$ structure

126. $v:=$ free variable

127. $s_{1}, s_{2}:=$ variable assignment functions into $\mathfrak{A}$

128. $\phi:=$ formula

\section{Satisfaction for all variable assignment func- tions}

129. $[1,2]$

130.

$$
(\forall s: \mathfrak{A} \models \sigma[s]) \underline{\vee}(\mathfrak{A} \models \sigma[s] \text { for no } s)
$$

131. $\mathcal{L}:=$ first-order language

132. $\mathfrak{A}:=\mathcal{L}$-structure

133. $\sigma:=$ sentence in $\mathcal{L}$

134. $s:=$ variable assignment function into $\mathfrak{A}$

\section{Model (formula)}

135. $[1,2]$

136. $(\mathfrak{A} \models \phi):=\mathfrak{A}$ is a model of $\phi$

137.

$$
\mathfrak{A} \models \phi \leftrightarrow \forall s: \mathfrak{A} \models \phi[s]
$$


138.

$$
\mathfrak{A} \models \Phi \leftrightarrow \forall \phi \in \Phi: \mathfrak{A} \models \phi
$$

139. $\phi:=$ formula in $\mathcal{L}$

140. $s:=$ variable assignment function into $\mathfrak{A}$

141. $\Phi:=$ set of $\mathcal{L}$-formulas

142. $\mathcal{L}:=$ first-order language

143. $\mathfrak{A}:=\mathcal{L}$-structure

\section{True Sentences}

144. $[1,2]$

145.

$$
\mathfrak{A} \models \sigma \leftrightarrow \forall s: \mathfrak{A} \models \sigma[s]
$$

146. $(\mathfrak{A} \models \sigma):=\mathfrak{A}$ is a model of $\sigma$

147. $\sigma:=$ sentence in $\mathcal{L}$

148. $\sigma$ is true in $\mathfrak{A}$

149. $s:=$ variable assignment function into $\mathfrak{A}$

150. $\Phi:=$ set of $\mathcal{L}$-formulas

151. $\mathcal{L}:=$ first-order language

152. $\mathfrak{A}:=\mathcal{L}$-structure 


\section{Satisfaction of formulas with the connective "and"}

153. $[1,2]$

154

$$
\mathfrak{A} \models(\alpha \wedge \beta)[s] \leftrightarrow \mathfrak{A} \models \alpha[s] \wedge \mathfrak{A} \models \beta[s]
$$

155. $(\alpha \wedge \beta) \equiv(\neg((\neg \alpha) \vee(\neg \beta)))$

156. (155) is an abbreviation.

157. $s:=$ variable assignment function into $\mathfrak{A}$

158. $\alpha[s], \beta[s]:=\mathcal{L}$-formulas with assignment function $s$

159. $\mathcal{L}:=$ first-order language

160. $\mathfrak{A}:=\mathcal{L}$-structure

\section{Satisfaction with the existential quantifier}

161. $[1,2]$

162.

$$
\mathfrak{A} \models(\exists x)(\alpha)[s] \leftrightarrow \exists a \in A: \mathfrak{A} \models \alpha[s[x \mid a]]
$$

163. $\mathfrak{A}:=$ structure

164. $A:=$ universe of $\mathfrak{A} ; \quad a \in A$

165. $x:=$ variable

166. $s[x \mid a](v):=x$-modification of the assignment function $s$ 167. $\alpha:=$ formula with the $x$-modification of the assignment function $s$ 


\section{Substitution into a Term}

168. $[1,2]$

169.

$$
u_{t}^{x} \text { ( } u \text { with } x \text { replaced by } t \text { ) if }
$$

(i) $(u$ is a variable not equal to $x) \rightarrow\left(u_{t}^{x}\right.$ is $\left.u\right)$

(ii) $(u$ is $x) \rightarrow\left(u_{t}^{x}\right.$ is $\left.t\right)$

(iii) $(u$ is a constant symbol $) \rightarrow\left(u_{t}^{x}\right.$ is $\left.u\right)$

(iv) $\left(u: \equiv f u_{1} u_{2} \ldots u_{n}\right) \rightarrow\left(u_{t}^{x}\right.$ is $\left.f\left(u_{1}\right)_{t}^{x}\left(u_{2}\right)_{t}^{x} \ldots\left(u_{n}\right)_{t}^{x}\right)$

170. $u, t, u_{t}^{x}, u_{i}:=$ terms

171. $x:=$ variable

172. $f:=n$-ary function

173. Note that in (169.iv), the parentheses have been added for the purpose of readability; so, $\left(u_{1}\right)_{t}^{x}: \equiv u_{1}^{x}$.

174. Substitution into a term (169) is a definition by recursion.

\section{Substitution into a Formula}

175. $[1,2]$

176.

$$
\phi_{t}^{x} \text { ( } \phi \text { with } x \text { replaced by } t \text { ) if }
$$

(i) $\left(\phi: \equiv=u_{1} u_{2}\right) \rightarrow\left(\phi_{t}^{x}\right.$ is $\left.=\left(u_{1}\right)_{t}^{x}\left(u_{2}\right)_{t}^{x}\right)$

(ii) $\left(\phi: \equiv R u_{1} u_{2} \ldots u_{n}\right) \rightarrow\left(\phi_{t}^{x}\right.$ is $\left.R\left(u_{1}\right)_{t}^{x}\left(u_{2}\right)_{t}^{x} \ldots\left(u_{n}\right)_{t}^{x}\right)$

(iii) $(\phi: \equiv \neg(\alpha)) \rightarrow\left(\phi_{t}^{x}\right.$ is $\left.\neg\left(\alpha_{t}^{x}\right)\right)$

(iv) $(\phi: \equiv(\alpha \vee \beta)) \rightarrow\left(\phi_{t}^{x}\right.$ is $\left.\left(\alpha_{t}^{x} \vee \beta_{t}^{x}\right)\right)$ 


$$
(v) \phi: \equiv(\forall y)(\alpha) \rightarrow \phi_{t}^{x}= \begin{cases}\phi, & \text { if } x \text { is } y \\ (\forall y)\left(\alpha_{t}^{x}\right), & \text { otherwise }\end{cases}
$$

177. $\mathcal{L}:=$ first-order language

178. $\phi, \phi_{t}^{x}:=\mathcal{L}$-formulas

179. $t:=$ term

180. $x:=$ variable

181. $R:=n$-ary relation

182. Note that in (176), the parentheses have been added for the purpose of readability; so, $\left(\phi_{1}\right)_{t}^{x}: \equiv \phi_{1 t}^{x}$.

183. Substitution into a formula (176) is a definition by recursion.

\section{A term substitutable for a variable in a for- mula}

184. $[1,2]$

185.

$t$ is substitutable for $x$ in $\phi$ if

(i) $\phi$ is atomic, or

(ii) $\phi: \equiv \neg(\alpha)$ and $t$ is substitutable for $x$ in $\alpha$, or

(iii) $\phi: \equiv(\alpha \vee \beta)$ and $t$ is substitutable for $x$ in both $\alpha$ and $\beta$, or (iv) $\phi: \equiv(\forall y)(\alpha)$ and either

(a) $x$ is not free in $\phi$, or

(b) $y$ does not occur in $t$ and $t$ is substitutable for $x$ in $\alpha$.

186. $\mathcal{L}:=$ first-order language 
187. $\phi, \alpha, \beta:=\mathcal{L}$-formulas

188. $t:=$ term

189. $x:=$ variable

190. Notice that

(i) certain operations are allowed only if $t$ is substitutable for $x$ in $\phi$

(ii) this restriction is important to preserve the truth of formulas after performing substitutions.

\section{Logical Implication (sets of formulas)}

191. $[1,2]$

192.

$$
(\forall \mathfrak{A}: \mathfrak{A} \models \Delta \rightarrow \mathfrak{A} \models \Gamma) \rightarrow(\Delta \models \Gamma)
$$

193. $(\Delta \models \Gamma):=\Delta$ logically implies $\Gamma$

194. $\mathcal{L}:=$ first-order language

195. $\mathfrak{A}:=\mathcal{L}$-structure

196. $\Delta, \Gamma:=$ sets of $\mathcal{L}$-formulas

197. (192) says that if $\Delta$ is true in $\mathfrak{A}$, then $\Gamma$ is true in $\mathfrak{A}$.

198. Recall that $\Delta$ is true in $\mathfrak{A}$ if $\forall s: \mathfrak{A} \models \Delta[s]$.

199. $s:=$ variable assignment function into $\mathfrak{A}$ 


\section{Valid Formulas}

200. $[1,2]$

201.

$$
(\models \phi) \rightarrow(\phi \text { is valid })
$$

202. $(\emptyset \models \phi): \equiv(\models \phi):=(\forall s: \phi$ is true $)$

203. $\mathcal{L}:=$ first-order language

204. $\phi:=\mathcal{L}$-formula

205. $s:=$ variable assignment function

206. Notice that

(i) $\mathfrak{A} \models \sigma$ means truth (if there is a structure on the left), whereas

(ii) $\Gamma \models \sigma$ means logical implication (if there is a set of sentences on the left).

207. $\mathfrak{A}:=\mathcal{L}$-structure

208. $\Gamma:=$ set of sentences in $\mathcal{L}$

209. $\sigma:=$ sentence

\section{Universal Closure of a Formula}

210. $[1,2]$

211.

$$
\models \phi \leftrightarrow \quad \models(\forall x)(\phi)
$$

212.

$(\phi$ has free variables $x, y, z) \rightarrow(\models \phi \leftrightarrow \models \forall x \forall y \forall z \phi)$

213. $\forall x \forall y \forall z \phi:=$ sentence called universal closure of $\phi$ 
214. $\mathcal{L}:=$ first-order language

215. $\phi:=\mathcal{L}$-formula

216. $x, y, z:=$ variables

\section{On the validity of a conditional statement of formulas}

217. $[1,2]$

218. $\models(\phi \rightarrow \psi) \rightarrow \phi \models \psi$

219. $\phi, \psi:=$ formulas

\section{"Bottom-up" Deduction}

220. $[1,2]$

221.

$$
(D: \equiv \Sigma \vdash \phi) \text { if } \forall i: 1 \leq i \leq n \text {, either }
$$

(i) $\phi_{i} \in \Lambda$, or

(ii) $\phi_{i} \in \Sigma$, or

(iii) $\exists\left(\Gamma, \phi_{i}\right): \Gamma \subseteq\left\{\phi_{1}, \phi_{2}, \ldots, \phi_{i-1}\right\}$.

222. $D: \equiv(\Sigma \vdash \phi):=$ deduction from $\Sigma$ of $\phi$

223. $\mathcal{L}:=$ first-order language

224. $\phi, \phi_{i}:=\mathcal{L}$-formulas

225. $\Lambda:=$ set of $\mathcal{L}$-formulas (logical axioms)

226. $\Sigma:=$ collection of $\mathcal{L}$-formulas (nonlogical axioms) 
227. $\left(\Gamma, \phi_{i}\right):=$ rule of inference

228. $D:=$ finite sequence $\left(\phi_{1}, \phi_{2}, \ldots, \phi_{n}\right)$ of $\mathcal{L}$-formulas

229. bottom-up := it defines a deduction in terms of its parts

\section{"Top-down" Deduction}

230. $[1,2]$

231.

$\mathrm{Thm}_{\Sigma}=\{\phi \mid \Sigma \vdash \phi\}$ is the smallest set $C$ such that

(i) $\Sigma \subseteq C$

(ii) $\Lambda \subseteq C$

(iii) $((\Gamma, \theta):=$ rule of inference $\wedge \Gamma \subseteq C) \rightarrow(\theta \in C)$

232. $\mathcal{L}:=$ first-order language

233. $\Sigma, \Lambda:=$ sets of $\mathcal{L}$-formulas

234. top-down := we can think of the collection of deductions from $\Sigma$ (called $\mathrm{Thm}_{\Sigma}$ ) as the closure of axioms under the application of the rules of inference.

\section{Decidable Set of Axioms}

235. $[1,2]$

236. decidable set of axioms $:=$ (we will be able to decide whether)

$$
\phi \in \Lambda \underline{\vee} \phi \notin \Lambda
$$

237. $\mathcal{L}:=$ first-order language

238. $\Lambda:=$ collection of logical axioms for $\mathcal{L}$ 


\section{(Non)Logical Axioms}

239. $[1,2]$

240.

$$
\Lambda \cup \Sigma:=\text { expanded set of axioms }
$$

241. $\mathcal{L}:=$ first-order language

242. $\Lambda:=$ collection of logical axioms for $\mathcal{L}$

243. $\Sigma:=$ collection of nonlogical axioms for $\mathcal{L}$

244. $\Lambda$ is fixed

245. The rules of inference are fixed.

246. $\Sigma$ must be specified for each deduction.

247. The collection $\Lambda$ of logical axioms is decidable.

248. nonlogical axioms := additional axioms, beyond the set of logical axioms

249. formula $:=($ axiom $) \underline{\vee}$ (arise from previous formulas in the deduction via a rule of inference)

\section{Equality Axioms}

250. $[1,2]$

251. (E1)

$$
x=x \text { for each variable } x
$$

252. (E2)

$$
\begin{array}{r}
{\left[\left(x_{1}=y_{1}\right) \wedge\left(x_{2}=y_{2}\right) \wedge \ldots \wedge\left(x_{n}=y_{n}\right)\right] \rightarrow} \\
\quad \rightarrow\left(f\left(x_{1}, x_{2}, \ldots, x_{n}\right)=f\left(y_{1}, y_{2}, \ldots, y_{n}\right)\right)
\end{array}
$$


253. (E3)

$$
\begin{aligned}
& {\left[\left(x_{1}=y_{1}\right) \wedge\left(x_{2}=y_{2}\right) \wedge \ldots \wedge\left(x_{n}=y_{n}\right)\right] \rightarrow} \\
& \quad \rightarrow\left(R\left(x_{1}, x_{2}, \ldots, x_{n}\right)=R\left(y_{1}, y_{2}, \ldots, y_{n}\right)\right)
\end{aligned}
$$

\section{Quantifier Axioms}

254. $[1,2]$

255. (Q1): Universal instantiation

$$
(\forall x \phi) \rightarrow \phi_{t}^{x} \text {, if } t \text { is substitutable for } x \text { in } \phi
$$

256. (Q2): Existential generalization

$$
\phi_{t}^{x} \rightarrow(\exists x \phi), \text { if } t \text { is substitutable for } x \text { in } \phi
$$

\section{Rules of Inference}

257. $[1,2]$

258. There are two types of rules of inference: propositional consequence and one dealing with quantifiers.

259. The set of rules of inference is decidable.

\section{Propositional Consequence: Definition}

260. $[1,2]$

261. If every truth assignment that makes each propositional formula in $\Gamma_{P}$ true also makes $\phi_{P}$ true, then $\phi_{P}$ is a propositional consequence of $\Gamma_{P}$.

262. $\Gamma_{P}:=$ set of propositional formulas 
263. $\phi_{P}:=$ propositional formula

264. Note that

$$
\left(\phi_{P}:=\text { tautology }\right) \leftrightarrow\left(\phi_{P} \text { is a propositional consequence of } \emptyset\right) \text {. }
$$

\section{Propositional Consequence: Tautology}

265. $[1,2]$

266.

$\left(\phi_{P}\right.$ is a propositional consequence of $\left.\Gamma_{P}\right) \leftrightarrow$ $\leftrightarrow\left(\left[\gamma_{1_{P}} \wedge \gamma_{2 P} \wedge \ldots \wedge \gamma_{n_{P}}\right] \rightarrow \phi_{P}\right)$ is a tautology

267. $\Gamma_{P}=\left\{\gamma_{1 P}, \gamma_{2 P}, \ldots, \gamma_{n P}\right\}:=$ nonempty finite set of propositional formulas

268. $\phi_{P}:=$ propositional formula

\section{Propositional Consequence: Extension to First- order Logic}

269. $[1,2]$

270.

\section{$\left(\phi_{P}\right.$ is a propositional consequence of $\left.\Gamma_{P}\right) \rightarrow$ \\ $\rightarrow(\phi$ is a propositional consequence of $\Gamma)$}

271. $\mathcal{L}:=$ first-order language

272. $\Gamma:=$ finite set of $\mathcal{L}$-formulas

273. $\phi:=\mathcal{L}$-formula 


\section{Rule of Inference of type (PC)}

274. $[1,2]$

275.

$\phi$ is a propositional consequence of $\Gamma \rightarrow$ $\rightarrow(\Gamma, \phi)$ is a rule of inference of type (PC)

276. $\mathcal{L}:=$ first-order language

277. $\Gamma:=$ finite set of $\mathcal{L}$-formulas

278. $\phi:=\mathcal{L}$-formula

\section{Rules of Inference of type (QR)}

279. $[1,2]$

280. Rules of inference of type (QR)
(i) $(\{\psi \rightarrow \phi\},(\forall x \phi))$
(ii) $(\{\phi \rightarrow \psi\},(\exists x \phi) \rightarrow \psi)$

281. $x:=$ variable (not free in $\psi$ )

282. $\psi, \phi:=$ formulas

283. (280) means if $x$ is not free in $\psi$ :

(i) from $\phi \rightarrow \psi$, it may be deduced $\psi \rightarrow(\forall x \phi)$;

(ii) from $\psi \rightarrow \phi$, it may be deduced $(\exists x \phi) \rightarrow \psi$. 


\section{On the validity and tautology of formulas}

284. $[1,2]$

285. ( $\theta$ is not valid $) \rightarrow\left(\theta_{P}\right.$ is not a tautology $)$

286. $\left(\theta_{P}\right.$ is tautology $) \rightarrow(\theta$ is a valid $)$

287. $\theta:=$ formula in first-order logic

288. $\theta_{P}:=$ formula in propositional logic

\section{List of requirements for axioms and rules of inference}

289. $[1,2]$

290. The following list is required for our axioms and rules of inference:

(i) There will be an algorithm that will decide, given a formula $\theta$, whether or not $\theta$ is a logical axiom.

(ii) There will be an algorithm that will decide, given a finite set of formulas $\Gamma$ and a formula $\theta$, whether or not $(\Gamma, \theta)$ is a rule of inference.

(iii) For each rule of inference $(\Gamma, \theta)$, $\Gamma$ will be a finite set of formulas.

(iv) Each logical axiom will be valid.

$(v)$ Our rules of inference will preserve truth. In other words, for each rule of inference $(\Gamma, \theta), \Gamma \models \theta$.

291. The requirements in (290) provide the basis of the Soundness Theorem.

\section{Logical Axioms: Valid}

292. $[1,2]$ 
293. Theorem: The logical axioms are valid.

\section{Rule of Inference: Theorem}

294. $[1,2]$

295. Theorem:

$$
(\Gamma, \theta):=\text { rule of inference } \rightarrow \Gamma \models \theta
$$

\section{Soundness Theorem}

296. $[1,2]$

297.

$$
\Sigma \vdash \phi \rightarrow \Sigma \models \phi
$$

298. $\mathcal{L}:=$ first-order language

299. $\Sigma:=$ set of $\mathcal{L}$-formulas

300. In words, the Soundness Theorem (297) tells us that in any structure $\mathfrak{A}$ that makes all of the formulas of $\Sigma$ true, $\phi$ is true as well.

301. If there is a deduction from $\Sigma$ of $\phi$, then $\Sigma$ logically implies $\phi$.

302. The purely syntactic notion of deduction is linked to the notions of truth and logical implication.

303. The Soundness Theorem is explicitly trying to relate the syntactical notion of deducibility $(\vdash)$ with the semantical notion of logical implication $(\models)$.

304. If there is a deduction of $\phi$ from $\Sigma$, then $\phi$ is true in any model of $\Sigma$. 


\section{When a variable is not free in a formula}

305. $[1,2]$

306.

$x$ is not free in $\psi \rightarrow(\phi \rightarrow \psi) \models[(\exists x \phi) \rightarrow \psi]$

307. $x:=$ variable

308. $\psi, \phi:=$ formulas

\section{Variable Assignment Functions and Substitu- tions}

309. $[1,2]$

310.

$$
s^{\prime}=s[x \mid \bar{s}(t)] \rightarrow \bar{s}\left(u_{t}^{x}\right)=\overline{s^{\prime}}(u)
$$

311. $u, t:=$ terms

312. $x:=$ variable

313. $s:$ Vars $\rightarrow A$

314. $s:=$ variable assignment function

315. $s[x \mid \bar{s}(t)]:=x$-modification of the assignment function $s$

316. $u_{t}^{x}:=u$ with $x$ replaced by $t$

\section{Term substitution in the $x$-modification of the assignment function}

317. $[1,2]$ 
318.

$$
\mathfrak{A} \models \phi_{t}^{x}[s] \leftrightarrow \mathfrak{A} \models \phi\left[s^{\prime}\right]
$$

319. $\mathcal{L}:=$ first-order language

320. $\phi:=$ formula

321. $x:=$ variable

322. $t:=$ term substitutable for $x$ in $\phi$

323. $s:$ Vars $\rightarrow A$

324. $s:=$ variable assignment function

325. $s^{\prime}=s[x \mid \bar{s}(t)]$

326. $s[x \mid \bar{s}(t)]:=x$-modification of the assignment function $s$

\section{Equality: Equivalence Relation}

327. $[1,2]$

328. Equality is an equivalence relation

$$
\begin{aligned}
& (\text { i }) \vdash x=x \\
& (\text { ii }) \vdash x=y \rightarrow y=x \\
& (\text { iii }) \vdash(x=y \wedge y=z) \rightarrow x=z
\end{aligned}
$$

\section{A set of formulas proves a formula if and only if it proves the formula for all variables}

329. $[1,2]$

330.

$$
\Sigma \vdash \theta \leftrightarrow \Sigma \vdash \forall x \theta
$$


331. For a formula to be true in a structure, it must be satisfied in that structure with every assignment function.

\section{Adding/deleting a universal quantifier}

332. $[1,2]$

333.

$$
\begin{array}{r}
\Sigma \vdash \theta \rightarrow\left(\Sigma^{\prime} \text { is formed by taking any } \sigma \in \Sigma\right. \text { and } \\
\text { adding or deleting a universal quantifier } \\
\text { whose scope is the entire formula } \left.\rightarrow \Sigma^{\prime} \vdash \theta\right)
\end{array}
$$

334. If we know $\Sigma \vdash \theta$, we can assume that every element of $\Sigma$ is a sentence: By quoting (333) several times, we can replace each $\sigma \in \Sigma$ with its universal closure.

\section{The Deduction Theorem}

335. $[1,2]$

336.

$$
(\Sigma \cup \theta \vdash \phi) \leftrightarrow(\Sigma \vdash(\theta \rightarrow \phi))
$$

337. $\theta:=$ sentence

338. $\Sigma:=$ set of formulas

339. The Deduction Theorem (336) says that there is a deduction of $\phi$ from the assumption $\theta$ if and only if there is a deduction of the implication $\theta \rightarrow \phi$.

340. In (336), we omit the braces of $\Sigma \cup\{\theta\} \vdash \phi$.

341. deduction $:=$ formal equivalents of the mathematical proofs 


\section{Proofs by Contradiction}

342. $[1,2]$

343.

$$
(\Sigma \vdash \eta) \leftrightarrow(\Sigma \cup(\neg \eta) \vdash[(\forall x) x=x] \wedge \neg[(\forall x) x=x])
$$

344. $\eta:=$ sentence

\section{Unary Relation Symbol}

345. $[1,2]$

346.

$$
\vdash[(\forall x) P(x)] \rightarrow[(\exists x) P(x)]
$$

347. $P:=$ unary relation symbol

\section{Binary Relation Symbol}

348. $[1,2]$

349.

$$
(\forall x)(\forall y) P(x, y) \vdash(\forall y)(\forall z) P(z, y)
$$

350. $P:=$ binary relation symbol

\section{Two unary relation symbols}

351. $[1,2]$

352.

$$
\vdash[(\forall x)(P(x)) \wedge(\forall x)(Q(x))] \rightarrow(\forall x)[P(x) \wedge Q(x)]
$$

353. $P, Q:=$ unary relation symbols 


\section{Complete Deductive System}

354. $[1,2]$

355.

$$
\forall \Sigma \forall \phi(\Sigma \models \phi \rightarrow \Sigma \vdash \phi) \rightarrow\left(\Lambda, \Gamma_{\theta}\right):=\text { complete }
$$

356. $\Lambda:=$ collection of logical axioms

357. $\Gamma_{\theta}:=$ collection of rules of inference

358. $\Sigma:=$ set of nonlogical axioms

359. $\mathcal{L}:=$ first-order language

360. $\phi:=\mathcal{L}$-formula

361. If $\phi$ is an $\mathcal{L}$-formula that is true in every model of $\Sigma$, then there will be a deduction from $\Sigma$ to $\phi$.

362. Our ability to prove $\phi$ depends on $\phi$ being true in every model of $\Sigma$.

\section{(In)Consistent}

363. $[1,2]$

364.

$$
\exists(\Sigma \vdash[(\forall x) x=x] \wedge \neg[(\forall x) x=x]) \rightarrow \Sigma \text { is inconsistent }
$$

365.

$\Sigma$ is not inconsistent $\rightarrow \Sigma$ is consistent

366. $\mathcal{L}:=$ first-order language

367. $\Sigma:=$ set of $\mathcal{L}$-formulas

$\Sigma$ proves a contradiction $\rightarrow \Sigma$ is inconsistent 
368.

$$
\Sigma \text { is inconsistent } \rightarrow \exists(\Sigma \vdash \phi)
$$

369. $\phi:=\mathcal{L}$-formula

370. $\phi:=[(\forall x) x=x] \wedge \neg[(\forall x) x=x]$

371. $\phi$ is a contradictory sentence $(\perp)$.

372. $\perp$ is a sentence that is false in every language and is true in no structure.

\section{Completeness Theorem}

373. $[1,2]$

374

$$
(\Sigma \models \phi) \rightarrow(\Sigma \vdash \phi)
$$

375. $\mathcal{L}:=$ first-order language

376. $\Sigma:=$ set of $\mathcal{L}$-formulas

377. $\phi:=\mathcal{L}$-formula

378. The Completeness Theorem finishes the link between deducibility and logical implication.

\section{Soundness + Completeness}

379. $[1,2]$

380.

$$
(\Sigma \models \phi) \leftrightarrow(\Sigma \vdash \phi)
$$

381. $\mathcal{L}:=$ first-order language 
382. $\Sigma:=$ set of $\mathcal{L}$-formulas

383. $\phi:=\mathcal{L}$-formula

\section{Compactness Theorem}

384. $[1,2]$

385.

$$
(\exists \mathfrak{A}: \mathfrak{A} \models \Sigma) \leftrightarrow\left(\forall \Sigma_{0} \exists \mathfrak{B}: \mathfrak{B} \models \Sigma_{0}\right)
$$

386. $\Sigma:=$ set of axioms

387. $(\mathfrak{A} \models \Sigma):=\mathfrak{A}$ is a model of $\Sigma$

388. $\Sigma_{0} \subseteq \Sigma$

389. $\Sigma_{0}:=$ finite subset of $\Sigma$

390. $\mathfrak{B}:=$ model of $\Sigma_{0}$

391. The Compactness Theorem

(i) is one use of the link between deducibility and logical implication;

(ii) focus our attention on the finiteness of deductions;

(iii) says that

$\Sigma$ is satisfiable $\leftrightarrow \Sigma$ is finitely satisfiable.

\section{(Finitely) Satisfiable}

392. $[1,2]$

393.

$$
(\exists \mathfrak{A}: \mathfrak{A} \models \Sigma) \rightarrow(\Sigma \text { is satisfiable })
$$


394.

$$
\left(\forall \Sigma_{0} \exists \mathfrak{B}: \mathfrak{B} \models \Sigma_{0}\right) \rightarrow(\Sigma \text { is finitely satisfiable })
$$

395. $\Sigma:=$ set of axioms

396. $(\mathfrak{A} \models \Sigma):=\mathfrak{A}$ is a model of $\Sigma$

397. $\Sigma_{0} \subseteq \Sigma$

398. $\Sigma_{0}:=$ finite subset of $\Sigma$

399. $\mathfrak{B}:=$ model of $\Sigma_{0}$

\section{Finite subset of a set of formulas}

400. $[1,2]$

401.

$$
(\Sigma \models \theta) \leftrightarrow\left(\exists \Sigma_{0} \subseteq \Sigma: \Sigma_{0} \models \theta\right)
$$

402. $\mathcal{L}:=$ first-order language

403. $\Sigma:=$ set of $\mathcal{L}$-formulas

404. $\theta:=\mathcal{L}$-formula

405. $\Sigma_{0}:=$ finite subset of $\Sigma$

\section{First-order Sentences: Natural Numbers}

406. $[1,2]$

407. No set of first-order sentences can completely characterize the structure of the natural numbers. 


\section{Theory of a Structure}

408. $[1,2]$

409.

$$
T h(\mathfrak{A})=\{\phi \mid \mathfrak{A} \models \phi\}
$$

410.

$$
T h(\mathfrak{A})=T h(\mathfrak{B}) \rightarrow \mathfrak{A} \equiv \mathfrak{B}
$$

411.

$$
(\mathfrak{A} \equiv \mathfrak{N}) \rightarrow(\mathfrak{A} \text { is a model of arithmetic })
$$

412. $\mathcal{L}:=$ first-order language

413. $\mathfrak{A}, \mathfrak{B}:=\mathcal{L}$-structures

414. $\phi:=\mathcal{L}$-formula

415. $(\mathfrak{A} \equiv \mathfrak{B}):=\mathfrak{A}$ and $\mathfrak{B}$ are elementarily equivalent

416. $\mathcal{L}_{N T}=\{0, S,+, \cdot, E,<\}$

417. $\mathcal{L}_{N T}:=$ language of number theory

418. $\mathfrak{N}:=\mathcal{L}_{N T}$-structure

\section{Substructure}

419. $[1,2]$

420. $\mathfrak{A} \subseteq \mathfrak{B}$ if

(i) $A \subseteq B$

(ii) $\forall c: c^{\mathfrak{A}}=c^{\mathfrak{B}}$

(iii) $\forall R: R^{\mathfrak{A}}=R^{\mathfrak{B}} \cap A^{n}$

(iv) $\forall f: f^{\mathfrak{A}}=f^{\mathfrak{B}} \uparrow_{A^{n}}$ 
421. (420.iv) means

$$
(\forall f)(\forall a \in A): f^{\mathfrak{A}}(a)=f^{\mathfrak{B}}(a) .
$$

422. $\mathcal{L}:=$ first-order language

423. $\mathfrak{A}, \mathfrak{B}:=\mathcal{L}$-structures

424. $(\mathfrak{A} \subseteq \mathfrak{B}):=\mathfrak{A}$ is a substructure of $\mathfrak{B}$

425. $A:=$ universe of $\mathfrak{A}$

426. $B:=$ universe of $\mathfrak{B}$

427. $R:=n$-ary relation symbol

428. $f:=n$-ary function symbol

429. $f^{\mathfrak{B}}\left\lceil_{A^{n}}:=\right.$ restriction of the function $f^{\mathfrak{B}}$ to the set $A^{n}$

430. A substructure of $\mathfrak{B}$ is completely determined by its universe, and this universe can be any nonempty subset of $B$ that contains the constants and is closed under every function $f$.

\section{Elementary Substructure/Extension}

431. $[1,2]$

432.

$(\mathfrak{A} \prec \mathfrak{B}):=\mathfrak{A}$ is an elementary substructure of $\mathfrak{B}$ (equivalently, $\mathfrak{B}$ is an elementary extension of $\mathfrak{A}$ ) if

$$
\forall s \forall \phi: \mathfrak{A} \models \phi[s] \leftrightarrow \mathfrak{B} \models \phi[s]
$$

433. $\mathcal{L}:=$ first-order language

434. $\mathfrak{A}, \mathfrak{B}:=\mathcal{L}$-structures 
435. $\mathfrak{A} \subseteq \mathfrak{B}$

436. $\phi:=\mathcal{L}$-formula

437. $s:$ Vars $\rightarrow A$

438. Vars $:=$ set of variables

439. $A:=$ universe of $\mathfrak{A}$

\section{Truth in elementary substructure/extension}

440. $[1,2]$

441.

$$
(\mathfrak{A} \prec \mathfrak{B}) \rightarrow(\sigma \text { is true in } \mathfrak{A} \leftrightarrow \sigma \text { is true in } \mathfrak{B})
$$

442. $\mathfrak{A}, \mathfrak{B}:=$ structures

443. $\sigma:=$ sentence

\section{Condition for an elementary substructure}

444. $[1,2]$

445.

$$
(\mathfrak{A} \subseteq \mathfrak{B}) \wedge(\forall \alpha \forall s: \mathfrak{B} \models \exists x \alpha[s], \exists a: \mathfrak{B} \models \alpha[s[x \mid a]]) \rightarrow(\mathfrak{A} \prec \mathfrak{B})
$$

446. $\mathfrak{A}, \mathfrak{B}:=$ structures

447. $\mathfrak{A} \subseteq \mathfrak{B}$

448. $\alpha:=$ formula

449. $s:$ Vars $\rightarrow A$

450. $A:=$ universe of $\mathfrak{A}$ 


\section{Hilbert Axiomatic System}

451. $[4,5]$

452. Hilbert-style calculus is performed in the Hilbert Axiomatic System, composed by 9 axioms and 1 rule (Modus Ponens).

453. rule $:=$ inference rule of logic

\section{Axioms of the Hilbert-style Calculus}

454. $[4,5]$

455. A, $B, C:=$ propositional variables or formulas

456. $\vdash A \rightarrow(B \rightarrow A)$

457. $\vdash(A \rightarrow(B \rightarrow C)) \rightarrow(A \rightarrow B) \rightarrow(A \rightarrow C)$

458. $\vdash(\neg A \rightarrow \neg B) \rightarrow B \rightarrow A$

459. $\vdash A \rightarrow(A \vee B)$

460. $\vdash A \rightarrow(B \vee A)$

461. $\vdash(A \rightarrow B) \rightarrow((C \rightarrow B) \rightarrow(A \vee C \rightarrow B))$

462. $\vdash(A \wedge B) \rightarrow A$

463. $\vdash(A \wedge B) \rightarrow B$

464. $\vdash A \rightarrow(B \rightarrow(A \wedge B))$

\section{Inference Rule of the Hilbert-style Calculus}

465. $[4,5]$ 
466. Modus Ponens

$$
\begin{aligned}
& \vdash P \\
& \vdash P \rightarrow Q \\
& \vdash Q
\end{aligned}
$$

\section{Sequent Systems: Classical Logic}

467. $[3,6]$

468. LK := sequent system for classical logic

469. sequents := basic syntactic units (finite sequence of formulas)

470. $\alpha_{i}, \beta_{i}:=$ formulas

471.

$$
\alpha_{1}, \ldots, \alpha_{m} \Rightarrow \beta_{1}, \ldots, \beta_{n}
$$

472. $m, n \geq 0$

473. (471) is a sequent.

474. $\Rightarrow$ is a sequent arrow.

475. $\alpha_{1}, \ldots, \alpha_{m}:=$ antecedents (conjunctive-like "assumptions")

476. $\beta_{1}, \ldots, \beta_{n}:=$ succedents (disjunctive-like "conclusions")

477. (471) means that $\left(\alpha_{1} \wedge \ldots \wedge \alpha_{m}\right)$ implies $\left(\beta_{1} \vee \ldots \vee \beta_{n}\right)$.

478.

$$
\alpha_{1}, \ldots, \alpha_{m} \Rightarrow
$$

means $\left(\alpha_{1} \wedge \ldots \wedge \alpha_{m}\right)$ leads to a contradiction.

479.

$$
\Rightarrow \beta_{1}, \ldots, \beta_{n}
$$

means $\left(\beta_{1} \vee \ldots \vee \beta_{n}\right)$ follows from no assumption. 
480. The provability of a sequent is a syntactical approach.

481. The validity of a sequent is a semantical approach.

482. A sequent system contains initial sequents (axiom schemes in Hilbertstyle systems) and rules.

483. rule $:=$ one/two upper sequents and one lower sequent

484. The lower sequent can be inferred from the upper sequents.

485.

$$
\frac{\text { upper sequents }}{\text { lower sequent }}
$$

486. $\Gamma, \Pi, \Delta, \ldots$ (capital Greek letters) := finite (possibly empty) sequences of formulas 
487. LK has three kinds of rules:

(i) (left/right) rules for $\vee, \wedge, \rightarrow, \neg$,

(ii) cut rule,

(iii) (left/right) structural rules.

488. The initial sequents are of the form $\alpha \Rightarrow \alpha$.

489. Rules for the logical connectives:

490.

$$
\frac{\alpha, \Gamma \Rightarrow \Pi \quad \beta, \Gamma \Rightarrow \Pi}{\alpha \vee \beta, \Gamma \Rightarrow \Pi}(\vee \mathrm{L})
$$

491.

$$
\frac{\Gamma \Rightarrow \Lambda, \alpha}{\Gamma \Rightarrow \Lambda, \alpha \vee \beta}(\mathrm{VR} 1) \quad \frac{\Gamma \Rightarrow \Lambda, \beta}{\Gamma \Rightarrow \Lambda, \alpha \vee \beta}(\mathrm{VR} 2)
$$

492.

$$
\frac{\alpha, \Gamma \Rightarrow \Pi}{\alpha \wedge \beta, \Gamma \Rightarrow \Pi}(\wedge \mathrm{L} 1) \quad \frac{\beta, \Gamma \Rightarrow \Pi}{\alpha \wedge \beta, \Gamma \Rightarrow \Pi}
$$

493.

$$
\frac{\Gamma \Rightarrow \Lambda, \alpha \quad \Gamma \Rightarrow \Lambda, \beta}{\Gamma \Rightarrow \Lambda, \alpha \wedge \beta}(\wedge \mathrm{R})
$$

494.

$$
\frac{\Gamma \Rightarrow \Lambda, \alpha \quad \beta, \Delta \Rightarrow \Pi}{\alpha \rightarrow \beta, \Gamma, \Delta \Rightarrow \Lambda, \Pi}(\rightarrow \mathrm{L}) \quad \frac{\alpha, \Gamma \Rightarrow \Lambda, \beta}{\Gamma \Rightarrow \Lambda, \alpha \rightarrow \beta}(\rightarrow \mathrm{R})
$$

495.

$$
\frac{\Gamma \Rightarrow \Lambda, \alpha}{\neg \alpha, \Gamma \Rightarrow \Lambda}(\neg \mathrm{L}) \quad \frac{\alpha, \Gamma \Rightarrow \Lambda}{\Gamma \Rightarrow \Lambda, \neg \alpha}(\neg \mathrm{R})
$$

496. Cut rule:

$$
\frac{\Gamma \Rightarrow \Lambda, \alpha \quad \alpha, \Delta \Rightarrow \Pi}{\Gamma, \Delta \Rightarrow \Lambda, \Pi}(\mathrm{cut})
$$


497. Structural rules:

(i) exchange rules

$$
\frac{\Gamma, \alpha, \beta, \Delta \Rightarrow \Pi}{\Gamma, \beta, \alpha, \Delta \Rightarrow \Pi}(\mathrm{eL}) \quad \frac{\Gamma \Rightarrow \Pi, \alpha, \beta, \Lambda}{\Gamma \Rightarrow \Pi, \beta, \alpha, \Lambda}(\mathrm{eR})
$$

(ii) contraction rules

$$
\frac{\alpha, \alpha, \Gamma \Rightarrow \Pi}{\alpha, \Gamma \Rightarrow \Pi}(\text { cont } \mathrm{L}) \quad \frac{\Gamma \Rightarrow \Pi, \alpha, \alpha}{\Gamma \Rightarrow \Pi, \alpha}(\text { cont R) }
$$

(iii) weakening rules

$$
\frac{\Gamma \Rightarrow \Pi}{\alpha, \Gamma \Rightarrow \Pi}(w \mathrm{~L}) \quad \frac{\Gamma \Rightarrow \Pi}{\Gamma \Rightarrow \Pi, \alpha}(w \mathrm{R})
$$

498. The parenthesis are labels for the rules.

499. Note that

$$
\frac{\Gamma \Rightarrow \Lambda, \alpha \quad \beta, \Delta \Rightarrow \Pi}{\alpha \rightarrow \beta, \Gamma, \Delta \Rightarrow \Lambda, \Pi}(\rightarrow \mathrm{L})
$$

in the special case where

$$
\Gamma=\alpha, \quad \Lambda=\Delta=\emptyset, \quad \Pi=\beta,
$$

the succedent is the Modus Ponens for the sequent arrow,

$$
\frac{\alpha \Rightarrow \alpha \quad \beta \Rightarrow \beta}{\alpha \rightarrow \beta, \alpha \Rightarrow \beta}(\rightarrow \mathrm{L}) \text {. }
$$

500. active formulas $:=$ formulas in the rules

501. cut formula := active formula of the cut rule

502. principal formula $:=$ formulas in lower sequents of the rules 503. side formulas := other formulas

504. left rules $:=(\# \Rightarrow)$ 
505. right rules $:=(\Rightarrow \#)$

506. When the upper sequent is provable, its lower sequent is also provable.

507. The structural rules control the order (exchange), duplication (contraction), and omission (weakening) of formulas in the cedents of a given sequent.

508. The left contraction rule means that each formula occurrence in the antecedents can be used more than once.

\section{Proofs and Provability (in LK)}

509. [3]

510.

$$
\begin{aligned}
\mathrm{P} & :=\text { proof }(\text { in } \mathrm{LK}) \text { of }(\Gamma \Rightarrow \Delta) \\
& :=\text { a finite tree-like figure defined inductively as follows }
\end{aligned}
$$

(i) every sequent in $\mathrm{P}$, except the initial sequents, is obtained by an application of any one of the rules,

(ii) $(\Gamma \Rightarrow \Delta):=$ end sequent of $\mathrm{P}$.

511. LK := sequent system for classical logic

512. $(\Gamma \Rightarrow \Delta):=$ sequent

513. end sequent $:=$ single lowest sequent

514.

$$
(\Gamma \Rightarrow \Delta \text { is provable in } \mathrm{LK}) \leftrightarrow(\text { there is a proof of } \Gamma \Rightarrow \Delta)
$$

515.

( $\alpha$ is provable in $\mathrm{LK}) \leftrightarrow(\Rightarrow \alpha$ is provable in $\mathrm{LK})$ 
516. $\alpha:=$ formula

517. $(\Rightarrow \alpha):=$ sequent

\section{Rules for single formulas (in LK)}

518. [3]

519. We will rewrite the rules of LK considering only single formulas in the sequents, instead of sequences of formulas, assuming that some sequences are empty.

520. LK := sequent system for classical logic

521. Rules for the logical connectives:

522.

$$
\frac{\alpha \Rightarrow \pi \quad \beta \Rightarrow \pi}{\alpha \vee \beta \Rightarrow \pi}(\mathrm{VL})
$$

523.

$$
\frac{\gamma \Rightarrow \alpha}{\gamma \Rightarrow \alpha \vee \beta}(\mathrm{VR} 1) \quad \frac{\gamma \Rightarrow \beta}{\gamma \Rightarrow \alpha \vee \beta}(\mathrm{VR} 2)
$$

524.

$$
\frac{\alpha \Rightarrow \pi}{\alpha \wedge \beta \Rightarrow \pi}(\wedge \mathrm{L} 1) \quad \frac{\beta \Rightarrow \pi}{\alpha \wedge \beta \Rightarrow \pi}(\wedge \mathrm{L} 2)
$$

525.

$$
\frac{\gamma \Rightarrow \alpha \quad \gamma \Rightarrow \beta}{\gamma \Rightarrow \alpha \wedge \beta}(\wedge \mathrm{R})
$$

526.

$$
\frac{\gamma \Rightarrow \alpha \quad \beta \Rightarrow \pi}{\alpha \rightarrow \beta, \gamma \Rightarrow \pi}(\rightarrow \mathrm{L}) \quad \frac{\alpha, \gamma \Rightarrow \beta}{\gamma \Rightarrow \alpha \rightarrow \beta}(\rightarrow \mathrm{R})
$$

527.

$$
\frac{\gamma \Rightarrow \lambda, \alpha}{\neg \alpha, \gamma \Rightarrow \lambda}(\neg \mathrm{L}) \quad \frac{\alpha, \gamma \Rightarrow \lambda}{\gamma \Rightarrow \lambda, \neg \alpha}(\neg \mathrm{R})
$$


528. Cut rule:

$$
\frac{\gamma \Rightarrow \alpha \quad \alpha \Rightarrow \pi}{\gamma \Rightarrow \pi}(\mathrm{cut})
$$

529. Structural rules:

(i) exchange rules

$$
\frac{\alpha, \beta \Rightarrow \pi}{\beta, \alpha \Rightarrow \pi}(\mathrm{eL}) \quad \frac{\gamma \Rightarrow \alpha, \beta}{\gamma \Rightarrow \beta, \alpha}(\mathrm{eR})
$$

(ii) contraction rules

$$
\frac{\alpha, \alpha \Rightarrow \pi}{\alpha \Rightarrow \pi} \text { (cont L) } \quad \frac{\gamma \Rightarrow \alpha, \alpha}{\gamma \Rightarrow \alpha} \text { (cont R) }
$$

(iii) weakening rules

$$
\frac{\gamma \Rightarrow \pi}{\alpha, \gamma \Rightarrow \pi}(w \mathrm{~L}) \quad \frac{\gamma \Rightarrow \pi}{\gamma \Rightarrow \pi, \alpha}(w \mathrm{R})
$$

\section{Multisets of Formulas}

530. [3]

531.

two multisets are distinguished from each other $\leftrightarrow$

$\leftrightarrow$ the multiplicity of any member of them is different

532.

$$
\left(\forall \Phi_{1}, \Phi_{2} \in S^{*}: \Phi_{1} \simeq \Phi_{2}\right) \leftrightarrow
$$

$\leftrightarrow\left(\forall s \in S:\right.$ multiplicity of $s$ in $\Phi_{1}=$ multiplicity of $s$ in $\left.\Phi_{2}\right)$

533. multiplicity := number of occurrences of any formula

534. $\{\alpha, \beta, \alpha\}=\{\beta, \alpha, \alpha\} \neq\{\alpha, \beta\}$

535. $S:=$ set of formulas; $S^{*}:=$ set of multisets 
536. $S^{*}:=$ all finite sequence of $s \in S$

537. $\simeq:=$ equivalence relation on $S^{*}$

538. $S^{*}=\left\{\Phi_{i} \mid \Phi_{i}:=\right.$ multiset $\}$

539. $\Phi_{1}, \Phi_{2}:=$ multisets

540.

$$
\left(M=S^{*} / \simeq\right) \rightarrow(M:=\text { the set of all finite multisets of } s \in S)
$$

541. $S^{*} / \simeq:=$ quotient set

\section{Logical constant 0}

542. [3]

543. $0:=$ falsum (falsehood) $:=$ arbitrary contradiction

544. $(\neg \alpha) \equiv(\alpha \rightarrow 0)$

545. $(0 \Rightarrow):=$ initial sequent meaning the falsum implies anything

\section{Orthologic}

546. $[7,8]$

547. orthologic (minimal quantum logic):= logic associated with the order relation of ortholattices

548.

$$
\mathcal{O}:=\text { ortholattice }:=\text { bounded lattice with } p^{\perp}
$$

549.

$$
\forall p \in \mathcal{O}: p \vee p^{\perp}=\top
$$


550. bounded lattice $:=$ lattice with smallest $(\perp)$ and biggest $(T)$ elements

551. lattice := poset such that every two elements have an infimum and a supremum

552. poset $:=$ partial ordered set

553. partial order $:=$ reflexive, transitive, and antisymmetric relation

554. $p^{\perp}:=$ orthocomplement (order-reversing involution $p \mapsto \neg p$ )

555. In particular, $\forall p, q \in \mathcal{O}$ :

$$
\begin{aligned}
p \leq q & \Rightarrow q^{\perp} \leq p^{\perp} \\
\neg p^{\perp} & =p \\
\neg \perp & =\top \\
\neg(p \vee q) & =p^{\perp} \wedge q^{\perp} \\
\neg(p \wedge q) & =p^{\perp} \vee q^{\perp} \\
p \wedge p^{\perp} & =\perp
\end{aligned}
$$

556. The other De Morgan's laws hold.

557. \# distributive law between $(\wedge, \vee)$

558. In the sequent calculus style the axiomatization of orthologic is sound and complete.

559. Axiomatization of Orthologic:

560.

$$
\overline{A \vdash A} \text { ax } \quad \frac{A \vdash B \quad B \vdash C}{A \vdash C} \text { cut }
$$

561.

$$
\overline{A \wedge B \vdash A} \wedge_{1} L \quad \overline{A \wedge B \vdash B} \wedge_{2} L \quad \frac{C \vdash A \quad C \vdash B}{C \vdash A \wedge B} \wedge R \quad \overline{C \vdash \top}^{\top} R
$$


562.

$$
\overline{A \vdash A \vee B} \vee_{1} R \quad \overline{B \vdash A \vee B} \vee_{2} R \quad \frac{A \vdash C \quad B \vdash C}{A \vee B \vdash C} \vee L \quad \overline{\perp \vdash C} \perp L
$$

563.

$$
\frac{A \vdash B}{\neg B \vdash \neg A} \neg \quad \overline{A \vdash \neg \neg A} \neg \neg R \quad \overline{\neg \neg A \vdash A} \neg \neg L \quad \overline{\top \vdash A \vee \neg A} \text { tnd }
$$

564. (560) $\sim \succ($ pre) order relation

565. (561) $\sim \succ$ bounded inf semi-lattice

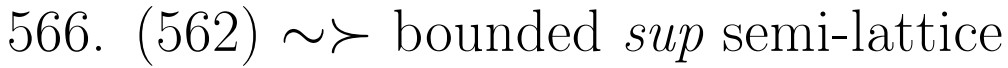

567. (563) $\sim \succ$ ingredients related to the orthocomplement $\neg A$

568. $(565)+(566) \sim \succ$ provides the structure of a bounded lattice

\section{Intuitionistic Reasoning}

569. [9]

570. $\neg A$ is an abbreviation for $A \rightarrow \perp$, i.e.,

$$
\neg A \equiv(A \rightarrow \perp) .
$$

571. Conjecture: Nothing is a proof of $\perp$ (falsity).

572. Many laws from classical logic are no longer valid due to the constructive meaning of the intuitionistic connectives.

573. The validity of $A \vee \neg A$ means there is a method to solve all mathematical problems.

574. There is a translation from classical formulas to intuitionistic ones. 
575. Classical propositional logic can be defined within the intuitionistic logic.

576. $\rightarrow, \wedge, \vee$ are all independent.

577. In intuitionistic propositional logic, an infinite number of non-equivalent formulas can be built from only one atomic formula $P[10]$.

578. Due to the intuitionistic refinement, equivalent formulas in classical propositional logic become no longer equivalent in intuitionistic propositional logic.

579. The intuitionistic logic has a richer language than the classical one.

580. Atomic formulas and connectives have a constructive interpretation.

\section{Intuitionistic Propositional Logic: Syntax}

581. [9]

582.

alphabet $:=$ consists of the following symbols:

(i) $P_{1}, P_{2}, P_{3}, \ldots:=$ atomic formulas or propositional variables [interpreted as (atomic) propositions]

(ii) $\rightarrow, \wedge, \vee, \neg:=$ connectives

(iii) $():,=$ brackets

583. Constructive interpretation of the connectives:

(i) $(A \rightarrow B):=$ one has a construction that transforms any proof of $A$ into a proof of $B$,

(ii) $(A \wedge B):=$ one can construct a proof of $A$ and one can construct a proof of $B$ 
(iii) $(A \vee B):=$ one has an algorithm that yields a proof of $A$ or a proof of $B$

(iv) $(\neg A):=(A \rightarrow \perp)$

(v) $\perp:=$ atomic formula (falsity)

584. A proof of $\perp$ implies a proof of any formula.

585. Formulas

$$
\begin{aligned}
& \text { (i) }\left(P:=P_{1} \vee P_{2} \vee P_{3} \underline{\vee} \ldots\right) \rightarrow(P:=\text { atomic formula }) \\
& \text { (ii) }(A, B:=\text { formulas }) \rightarrow((A \rightarrow B),(A \wedge B),(A \vee B),(\neg A):= \\
& \text { composite formulas })
\end{aligned}
$$

586. $\underline{\vee}$ is the exclusive or.

\section{Axiom Schema for Intuitionistic Propositional Logic}

587. [9]

588. There are ten axioms and one rule in the intuitionistic propositional logic, which is obtained by replacing the axiom $\neg \neg A \rightarrow A$ of classical logic by $\neg A \rightarrow(A \rightarrow B)$.

589. Axioms :

590. $A \rightarrow(B \rightarrow A)$

591. $(A \rightarrow B) \rightarrow((A \rightarrow(B \rightarrow C)) \rightarrow(A \rightarrow C))$

592. $A \rightarrow(B \rightarrow A \wedge B)$

593. $A \wedge B \rightarrow A$

594. $A \wedge B \rightarrow B$ 
595. $A \rightarrow A \vee B$

596. $B \rightarrow A \vee B$

597. $(A \rightarrow C) \rightarrow((B \rightarrow C) \rightarrow(A \vee B \rightarrow C))$

598. $(A \rightarrow B) \rightarrow((A \rightarrow \neg B) \rightarrow \neg A)$

599. $\neg A \rightarrow(A \rightarrow B)$

600. Rule of inference: (Modus Ponens)

$$
A, A \rightarrow B \vdash B .
$$

\section{Modal operators}

601. [3]

602. $\square, \diamond:=$ (unary) modal operators

603. $\diamond \varphi \equiv \neg \square \neg \varphi$

604. $\square$ can be interpreted as necessarily.

605. $\diamond$ can be interpreted as possibly.

606. $\varphi:=$ formula

\section{Decision problem}

607. [16]

608. In computability theory and computational complexity theory, a decision problem is a problem that can be posed as a yes-no question of the input values.

609. An example of a decision problem is deciding whether a given natural number is prime. 
610. A decision problem which can be solved by an algorithm is called decidable.

611.

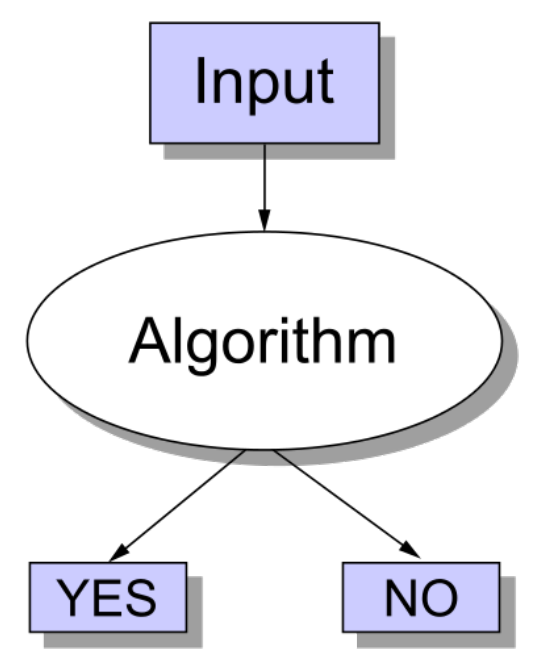

\section{Undecidable}

612. [15]

613. In computability theory and computational complexity theory, an undecidable problem is a decision problem for which it is proved to be impossible to construct an algorithm that always leads to a correct yes-or-no answer.

614. The halting problem is an example: it can be proven that there is no algorithm that correctly determines whether arbitrary programs eventually halt when run.

\section{Word problem}

615. [17] 
616. In mathematics and computer science, a word problem for a set $S$ with respect to a system of finite encodings of its elements is the algorithmic problem of deciding whether two given representatives represent the same element of the set.

617. The problem is commonly encountered in abstract algebra, where given a presentation of an algebraic structure by generators and relators, the problem is to determine if two expressions represent the same element; a prototypical example is the word problem for groups.

618. Less formally, the word problem in an algebra is: given a set of identities $E$, and two expressions $x$ and $y$, is it possible to transform $x$ into $y$ using the identities in $E$ as rewriting rules in both directions?

619. While answering the question in (618) may not seem hard, the remarkable (and deep) result that emerges, in many important cases, is that the problem is undecidable.

620. Many, if not most all, undecidable problems in mathematics can be posed as word problems.

621. List of undecidable problems https://en.wikipedia.org/wiki/List_of_undecidable_problems

\section{Natural Deduction in Heyting Semantics}

622. $[19,20]$

623. rules of natural deduction + Heyting Semantics $\sim \succ$ special way of constructing functions

624. $A, B, B_{i}:=$ formulas

625. formula $A:=$ set of its possible deductions; e.g., if $A=\{\alpha, \beta\}$ then both $\alpha$ and $\beta$ prove $A$ 
626. hypotheses $B_{i} \in A$

627. $\left(B_{1}, \ldots, B_{n} \vdash A\right) \equiv t\left[x_{1}, \ldots, x_{n}\right]: B_{1} \times \ldots \times B_{n} \rightarrow A$

628. $x_{i}:=$ variables

629. Two occurrences of the same formula $B_{i}$ in the same parcel of hypotheses correspond to the same variable.

630. The rules

(i) Hypothesis: $A$

(ii) Introductions:

$[A]$

$$
\frac{A \quad B}{A \wedge B} \wedge \mathcal{I} \quad \frac{B}{A \rightarrow B} \rightarrow \mathcal{I} x \quad \frac{A}{\forall x \cdot A} \forall \mathcal{I} \quad \frac{A[a / x]}{\exists x \cdot A} \exists \mathcal{I}
$$

$[A]$

$$
\frac{A}{A \vee B} \vee 1 \mathcal{I} \quad \frac{B}{A \vee B} \vee 2 \mathcal{I} \quad \begin{gathered}
\vdots \\
\frac{\perp}{\neg A} \neg \mathcal{I}
\end{gathered}
$$

$$
\begin{array}{cc}
{[A]} & {[B]} \\
\vdots & \vdots \\
B & A \\
\hline A \leftrightarrow & B
\end{array}
$$

(iii) Eliminations:

$$
\begin{aligned}
& \frac{A \wedge B}{A} \wedge 1 \mathcal{E} \\
& \frac{A \wedge B}{B} \wedge 2 \mathcal{E} \\
& \frac{A \rightarrow B \quad A}{B} \rightarrow \mathcal{E} \\
& \text { [A] } \\
& \frac{\exists x . A \quad{ }^{B}}{B} \exists \mathcal{E} \quad \quad \quad \frac{\forall x \cdot A}{A[a / x]} \forall \mathcal{E}
\end{aligned}
$$


$[A] \quad[B]$

$$
\begin{aligned}
& \frac{A \vee B \quad C \quad C}{C} \vee \mathcal{E} \quad \frac{\neg A \quad A}{\perp} \neg \mathcal{E} \\
& \frac{A \leftrightarrow B \quad A}{B} \leftrightarrow \mathcal{E} 1 \quad \frac{A \leftrightarrow B \quad B}{A} \leftrightarrow \mathcal{E} 2
\end{aligned}
$$

(iv) Absurdity:

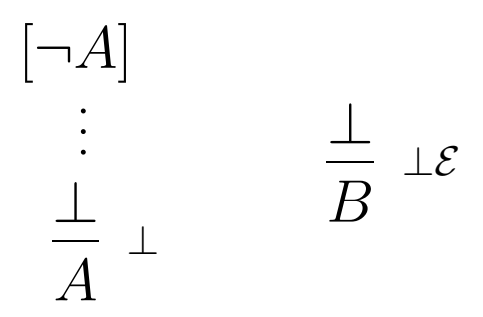

631. In $\exists \mathcal{E}, x$ cannot be free in $B$ and in any hypothesis that has not being canceled, except in $A$, in the deduction of $B$.

632. In $\forall \mathcal{I}, x$ cannot be free in any hypothesis that has not being canceled in the deduction of $A$.

633. In (630), $a$ is free for $x$ in $A$.

634. The left deduction of (630.iv) is called reductio ad absurdum.

635. The fingerprint of classical logic is the reductio ad absurdum.

636. Interpretation of the rules

$$
\begin{aligned}
& \text { (i) } \exists ! B_{1}: B_{1} \vdash A \Rightarrow x \equiv\left(B_{1} \vdash A\right) \Rightarrow x \in B_{1} \in A \\
& \text { (ii) }\left(u\left[x_{1}, \ldots, x_{n}\right]: A\right) \wedge\left(v\left[x_{1}, \ldots, x_{n}\right]: B\right) \Rightarrow \\
& \quad \Rightarrow\left\langle u\left[x_{1}, \ldots, x_{n}\right], v\left[x_{1}, \ldots, x_{n}\right]\right\rangle: A \wedge B
\end{aligned}
$$

(note that $u$ and $v$ have been made to depend on the same variables; their choices are correlated) 


$$
\begin{array}{cccc}
\vdots & \vdots & \vdots & \vdots \\
u: A & \overline{v: B} & \frac{u: A}{\langle u, v\rangle: A \wedge B}
\end{array}
$$

(iii) $t\left[x_{1}, \ldots, x_{n}\right]: A \wedge B \Rightarrow \pi^{1} t\left[x_{1}, \ldots, x_{n}\right]: A$

$t:=$ proof of a conjunction

$\pi^{1} t:=$ first projection

$\pi^{2} t: B$

$\pi^{2} t:=$ second projection

$$
\overline{t: A \wedge B} \quad \frac{t: A \wedge B}{\pi^{1} t: A} \quad \frac{t: A \wedge B}{\pi^{2} t: B}
$$

The following equations are the essence of the correspondence between logic and computer science:

$$
\begin{gathered}
\pi^{1}\langle u, v\rangle=u ; \quad \pi^{2}\langle u, v\rangle=v ; \quad\left\langle\pi^{1} t, \pi^{2} t\right\rangle=t . \\
\vdots \quad \vdots \\
\frac{u: A \quad v: B}{\langle u, v\rangle: A \wedge B} \frac{\vdots}{u: A}
\end{gathered} \frac{\overline{u: A}}{}
$$

$$
\frac{\frac{u: A \quad v: B}{\langle u, v\rangle: A \wedge B}}{\pi^{2}\langle u, v\rangle: A} \quad \bar{\vdots}
$$


(iv) $\lambda x . v$ is a function from $A$ to $B$ with $v\left[a, x_{1}, \ldots, x_{n}\right] \in V, a \in A$ (in $\lambda x . v\left[x, x_{1}, \ldots, x_{n}\right], x$ is bound)

(note that binding corresponds to discharge)

$$
\begin{gathered}
{[x: A]} \\
\vdots \\
v: B \\
\hline \lambda x . v: A \rightarrow B
\end{gathered}
$$

(v) $\left(t\left[x_{1}, \ldots, x_{n}\right]: A \rightarrow B\right) \wedge\left(u\left[x_{1}, \ldots, x_{n}\right]: A\right) \Rightarrow$

$\Rightarrow t\left[x_{1}, \ldots, x_{n}\right] u\left[x_{1}, \ldots, x_{n}\right]: B$

$t: A \rightarrow B$ for fixed values of $x_{1}, \ldots, x_{n}$

$u \in A ; \quad t(u) \in B$

$$
\frac{t: A \rightarrow B \quad u: A}{t u: B}
$$

We have:

$$
\begin{aligned}
(\lambda x . v) u & =v[u / x], \\
\lambda x . t x & =t \quad(\text { when } x \text { is not free in } t) .
\end{aligned}
$$

$$
\begin{gathered}
{[x: A]} \\
\vdots \\
v: B \\
\hline \lambda x \cdot v: A \rightarrow B
\end{gathered}
$$

$$
\frac{t: A \rightarrow B \quad u: A}{v: B}
$$$$
\overline{v[u / x]: B}
$$

637. In natural deduction, a proof is normal if it does not contain any sequence of an introduction and an elimination rule. (menemonic rule: $\mathrm{Nn}_{i e}$ )

\section{Lambda Calculus: Types}

638.

[19] 
639. In Heyting's approach, formulas become types.

640. The only types are the following:

(i) $T_{1}, \ldots, T_{n}:=$ atomic types $:=$ types;

(ii) $(U, V:=$ types $) \Rightarrow(U \times V, U \rightarrow V:=$ types $)$.

\section{Lambda Calculus: Terms}

641. [19]

642. Proofs become terms.

643. mnemonic rule: $\left(\mathrm{ft}_{y} \cdot \mathrm{pt} \mathrm{t}_{e}\right) \equiv($ formulas $\sim \succ$ types, proofs $\sim \succ$ terms)

644. term of type $A:=$ proof of a formula $A$

645. $x_{0}^{T}, \ldots, x_{n}^{T}, \ldots:=$ terms of type $T$

646. $(u, v:=$ terms of types $U$ and $V) \rightarrow(\langle u, v\rangle:=$ term of type $U \times V)$

647. ( $t:=$ term of type $U \times V) \rightarrow\left(\pi^{1} t, \pi^{2} t:=\right.$ terms of types $U$ and $V$, respectively)

648. $\left((v:=\right.$ term of type $V) \wedge\left(x_{n}^{U}:=\right.$ variable of type $\left.\left.U\right)\right) \rightarrow$ $\rightarrow\left(\lambda x_{n}^{U} \cdot v:=\right.$ term of type $\left.U \rightarrow V\right)$

649.

$$
\begin{gathered}
{\left[x_{n}^{U} \in U\right]} \\
\vdots \\
v \in V \\
\lambda x_{n}^{U} \cdot v \in U \rightarrow V
\end{gathered}
$$

650. ( $t, u:=$ terms of type $U \rightarrow V$ and $U$, respectively $) \rightarrow$

$\rightarrow(t u:=$ term of type $V)$ 


\section{Lambda Calculus: Denotational significance}

651. [19]

652. (object of type $U \rightarrow V) \equiv$ (function $f: U \rightarrow V$ )

653. (object of type $U \times V) \equiv$ (ordered pair $\langle u, v\rangle, u \in U$ and $v \in V$ )

654. $x^{T}:=$ variable of type $T$

655. $\langle u, v\rangle:=$ ordered pair

656. $\pi^{1} t:=$ first projection of $t$

657. $\pi^{2} t:=$ second projection of $t$

658. $\lambda x^{U} \cdot v: U \rightarrow V$ such that $\lambda x^{U} \cdot v[u]=v[u / x]$ with $x^{U} \equiv u$

659. $u:=$ object of type $U$

660. $t u:=$ function $t$ applied to the argument $u$

661. The following are primary equations:

$$
\begin{aligned}
\pi^{1}\langle u, v\rangle & =u, \\
\pi^{2}\langle u, v\rangle & =v, \\
\left(\lambda x^{U} \cdot v\right) u & =v[u / x] .
\end{aligned}
$$

662. The following are secondary equations:

$$
\begin{aligned}
\left\langle\pi^{1} t, \pi^{2} t\right\rangle & =t, \\
\lambda x^{U} \cdot t x & =t \quad(x \text { not free in } t) .
\end{aligned}
$$

\section{System of equations in lambda calculus: Consistent and decidable}

663.

[19] 
664. Theorem. The system given by (661) and (662) is consistent and decidable.

665. Consistency means that $x=y$, where $x$ and $y$ are distinct variables, cannot be proved.

\section{Conversion}

666. [19]

667. $t, t^{\prime}:=$ terms

668. In natural deduction, a proof is normal if it does not contain any sequence of an introduction and an elimination rule.

(menemonic rule: $\mathrm{Nn}_{i e}$ )

669. $\left(\lambda x^{U} \cdot v\right) u \sim \succ$ introduction

670. $\left\{\pi^{1}\langle u, v\rangle, \pi^{2}\langle u, v\rangle\right\} \sim \succ$ elimination

671. none subterms are of the form $\left(\lambda x^{U} . v\right) u$ or $\pi^{1}\langle u, v\rangle$ or $\pi^{2}\langle u, v\rangle \Rightarrow$ $\Rightarrow$ term $:=$ normal form

672. $t$ converts to $t^{\prime}$ if either:

(i) $t=\pi^{1}\langle u, v\rangle, t^{\prime}=u$; or

(ii) $t=\pi^{2}\langle u, v\rangle, t^{\prime}=v$; or

(iii) $t=\left(\lambda x^{U} \cdot v\right) u, t^{\prime}=v[u / x]$.

$$
\begin{gathered}
{\left[x^{U} \in U\right]} \\
\vdots \\
v \in V \\
\frac{v x^{U} . v}{} \in U \rightarrow V
\end{gathered}
$$

673. $t:=$ redex

674. $t^{\prime}:=$ contractum 
675. $t$ and $t^{\prime}$ are of the same type

676. $\exists$ sequence $u=t_{0}, t_{1}, \ldots, t_{n-1}, t_{n}=v$ : for $i=0,1, \ldots, n-1$,

$t_{i+1}$ is obtained from $t_{i}$ by replacing a redex by its contractum $\Rightarrow$

$\Rightarrow u \rightsquigarrow v$

677. $(u \rightsquigarrow v):=u$ reduces to $v$

678. $\rightsquigarrow$ is reflexive and transitive.

679. $((t \rightsquigarrow u) \wedge(u:=$ normal $)) \equiv(\exists ! u: u:=$ normal form for $t)$

680. $(t:=$ normal $) \leftrightarrow t$ is in head normal form $\left(\lambda x_{1} x_{2} \ldots x_{n} . y u_{1} u_{2} \ldots u_{m}\right)$ (where $y=x_{i} \vee \underline{\vee} y \neq x_{i}, u_{j}$ are normal)

681. A term converts in one step, reduces in many.

682. Conversion can be identified as rewriting, the left member being rewritten to the right one.

\section{The Curry-Howard Isomorphism}

683. [18-20]

684. This is an isomorphism between proofs and functional terms.

685. variable $x_{i}^{A} \equiv \operatorname{deduction} A(A$ in parcel $i)$

686. Recall the following rules for natural deduction

(i) Hypothesis: $x: A$

(ii) Introductions:

$$
\frac{x: A \quad y: B}{x y: A \wedge B} \wedge \mathcal{I} \quad \begin{gathered}
{[x: A]} \\
\frac{y: B}{\lambda x . x y: A \rightarrow B} \rightarrow \mathcal{I x}
\end{gathered} \quad \frac{x: A}{\forall \xi \cdot A} \forall \mathcal{I} \quad \frac{A[a / \xi]}{\exists \xi \cdot A} \exists \mathcal{I}
$$




$$
\begin{aligned}
& \frac{x: A}{A \vee B} \vee 1 \mathcal{I} \quad \frac{y: B}{A \vee B} \vee 2 \mathcal{I} \\
& {[x: A]} \\
& \frac{\perp}{\neg A} \neg \mathcal{I}
\end{aligned}
$$

$$
\begin{array}{cc}
{[A]} & {[B]} \\
\vdots & \vdots \\
B & A \\
\hline A \leftrightarrow B
\end{array} \leftrightarrow \mathcal{I}
$$

(iii) Eliminations:

$$
\begin{aligned}
& \frac{x y: A \wedge B}{x: A} \wedge 1 \mathcal{E} \quad \frac{x y: A \wedge B}{y: B} \wedge 2 \mathcal{E} \quad \frac{\lambda x . x y: A \rightarrow B \quad x: A}{y: B} \rightarrow \mathcal{E} \\
& \text { [A] } \\
& \frac{\exists x . A \quad B}{B} \exists \mathcal{E} \quad \frac{\forall \xi \cdot A}{A[a / \xi]} \forall \mathcal{E} \\
& {[x: A] \quad[y: B]}
\end{aligned}
$$

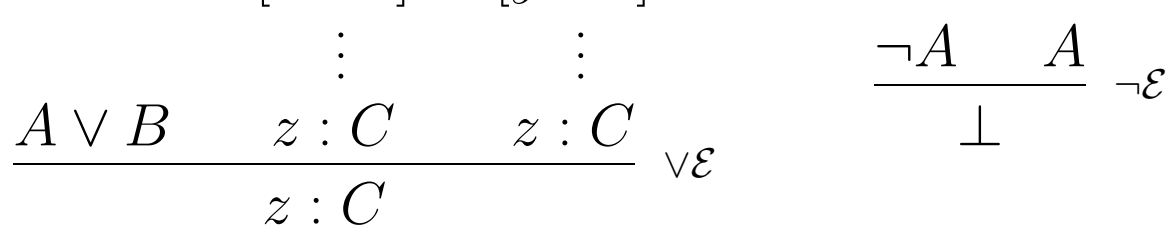

$$
\begin{aligned}
& \frac{A \leftrightarrow B \quad A}{B} \leftrightarrow \mathcal{E} 1 \quad \frac{A \leftrightarrow B \quad B}{A} \leftrightarrow \mathcal{E} 2
\end{aligned}
$$

(iv) Absurdity:

$$
\begin{gathered}
{[\neg A]} \\
\vdots \\
\frac{\perp}{x: A} \perp
\end{gathered}
$$

687.

$$
\begin{array}{cccc}
\vdots & \vdots & \vdots & \vdots \\
u: A & \overline{v: B} & \frac{u: A}{\langle u, v\rangle: A \wedge B}
\end{array} \wedge \mathcal{I}
$$


688.

$$
\begin{array}{ccc}
\vdots & \vdots & \vdots \\
t: A \wedge B & \frac{t: A \wedge B}{\pi^{1} t: A} \wedge 1 \mathcal{E} & \frac{t: A \wedge B}{\pi^{2} t: B} \wedge 2 \mathcal{E}
\end{array}
$$

689. if the deleted hypotheses form parcel $i$

$$
\begin{aligned}
& {\left[x_{i}: A\right]} \\
& \frac{v: B}{\lambda x_{i}^{A} \cdot v: A \rightarrow B} \rightarrow \mathcal{I} x_{i}
\end{aligned}
$$

690. term $t u$

$$
\frac{t: A \rightarrow B \quad u: A}{t u: B} \rightarrow \mathcal{E}
$$

691. Conversion, normality, and reduction correspond perfectly on both sides of the isomorphism. (mnemonic: cnr.iso)

\section{The Normalization Theorem}

692. [19]

693. typed $\lambda$-calculus $\sim \succ$ behaves well computationally

694. Normalization Theorem $\sim \succ$ existence (normal form)

695. Church-Rosser property $\sim \succ$ uniqueness (normal form)

696. mnemonic: $\mathrm{NeCRu}$

697. (694) $\sim \succ$ two forms: 
(i) weak $\sim \succ \exists$ terminating strategy (normalization)

(ii) strong $\sim \succ$ all possible strategies (normalization) terminate

\section{The lambda-calculus: Introduction}

698. [21]

699. $\lambda$-calculus $\sim \succ$ collection of several formal systems

700. Example:

701. $f(x)=x-y ; \quad g(y)=x-y$

702. $f: x \mapsto x-y ; \quad g: y \mapsto x-y$

703. $f=\lambda x \cdot x-y ; \quad g=\lambda y \cdot x-y$

704. $f(0)=0-y ; \quad f(1)=1-y$

705. $(\lambda x \cdot x-y)(0)=0-y ; \quad(\lambda x \cdot x-y)(1)=1-y$

\section{The lambda-calculus: Formal system}

706. [21]

707. $\lambda$-term $:=$ atom $\underline{\vee}$ application $\underline{\vee}$ abstraction
(a) $v_{i}, c_{i}:=\lambda$-terms (atoms)
(b) $(M, N:=\lambda$-terms $) \rightarrow((M N):=\lambda$-term (application)
(c) $(M:=\lambda$-term $\wedge x:=$ variable $) \rightarrow$
$\rightarrow((\lambda x \cdot M):=\lambda$-term (abstraction) $)$

708. $v_{i}:=$ variables

709. $c_{i}:=$ atomic constants 
710. $x, y, z:=$ distinct variables $\Rightarrow M=y z \Rightarrow(\lambda x \cdot M)=(\lambda x \cdot(y z)):=$ vacuous abstraction $(x$ does not occur in $M):=$ constant functions

711. $\lambda$ and $\lambda x$ are not terms.

712. $M, N, P, Q, \ldots:=\lambda$-terms

713. $x, y, z, u, v, w, \ldots:=$ variables

714. $M \equiv N$ means syntactic identity, i.e., $M$ is exactly the same term as $N$.

715. Application: $M N P Q \equiv(((M N) P) Q)$

(association from left to right)

716. $\lambda x \cdot P Q \equiv(\lambda x .(P Q))$

717. Abstraction: $\lambda x_{1} x_{2} \ldots x_{n} \cdot M \equiv\left(\lambda x_{1} \cdot\left(\lambda x_{2} \cdot\left(\ldots\left(\lambda x_{n} \cdot M\right)\right)\right)\right)$

(from right to left)

718. menemonic: app.lr, abs.rl

719. $(M N \equiv P Q) \rightarrow(M \equiv P \wedge N \equiv Q)$

720. $(\lambda x . M \equiv \lambda y \cdot P) \rightarrow(x \equiv y \wedge M \equiv P)$

721. $k=0$ in $P \equiv M N_{1} \ldots N_{k}(k \geq 0)$ means $P \equiv M$.

722. $n=0$ in $\lambda x_{1} \ldots x_{n} . P Q$ means $P Q$.

723. $\lambda:=$ (abbreviated as) $\lambda$-calculus in general

724. iff $:=$ if and only if

\section{The lambda-calculus: Informal interpretation}

725. [21]

726. $(M:=$ function/operator $) \Rightarrow(M N:=\operatorname{application}$ of $M$ to $N)$ 
727. $(\lambda x \cdot M)(N):=$ operator/function substituting $N$ for $x$ in $M$

728. $x y:=$ application

729. $\lambda x \cdot x(x y):=$ the operation of applying a function twice to $y$

730. $(\lambda x . x(x y))(N)=N(N y)$ holds for all terms $N$.

731. $\lambda x \cdot y:=$ constant function (value $y$ for all arguments)

732. $(\lambda x . y) N=y$

\section{Lambda-terms: Length, occurrence, scope, free and bound variables, substitution}

733. [21]

734.

$\operatorname{lgh}(M):=$ total number of occurences of $c_{i}, v_{i}$ in $M$

(a) $\operatorname{lgh}(a)=1$

(b) $\operatorname{lgh}(M N)=\operatorname{lgh}(M)+\operatorname{lgh}(N)$

(c) $\operatorname{lgh}(\lambda x \cdot M)=1+\operatorname{lgh}(M)$

735. $\operatorname{lgh}(M):=$ length of $M$

736. $M, N, P, Q:=\lambda$-terms

737. $c_{i}, v_{i}, a, x:=\lambda$-terms (atoms)

738. $x, y, z, u, v, v_{i}:=$ variables

739. induction on $M \equiv$ induction on $\operatorname{lgh}(M)$

740. e.g., $M \equiv x y z(\lambda x y . u v) \rightarrow \operatorname{lgh}(M)=7$ 
741.

$P$ occurs in $Q \equiv P$ is a subterm of $Q \equiv Q$ contains $P$ (relation defined by induction on $Q$ )

(a) $P$ occurs in $P$

(b) $(P$ occurs in $M) \vee(P$ occurs in $N) \rightarrow(P$ occurs in $M N)$

(c) $(P$ occurs in $M) \vee(P \equiv x) \rightarrow(P$ occurs in $\lambda x . M)$

742. In $z(\lambda y .(x y z))$ there are two occurences of $z$ and $y$, and one occurrence of $x$.

743. In $\lambda x . M, M$ is the scope of $\lambda x$.

744. (i) $(x \in M$ in $\lambda x . M) \rightarrow(x$ is bound $)$

(ii) the $x$ in $\lambda x$ is bound and binding

(iii) $x$ is free otherwise

745. In $x \lambda x . x$, the left $x$ is a free variable and the right $x$ is a bound variable.

746. $\mathrm{FV}(P):=$ set of all free variables of $P$

747. closed term $:=$ a term with no free variables

748.

$$
[N / x] M:=\text { substitution of } N, \forall x^{f} \in M
$$

749. $x^{f}:=$ free occurrence of $x$

750. The definition of substitution is by induction on $M$ :

(let $x \not \equiv y$ and $z \notin \mathrm{FV}(N P)$ )
(a) $[N / x] x \equiv N$
(b) $[N / x] a \equiv a, \forall a \not \equiv x$
(c) $[N / x](P Q) \equiv([N / x] P[N / x] Q)$
(d) $[N / x](\lambda x . P) \equiv \lambda x . P$ 

(e) $x \notin \mathrm{FV}(P) \rightarrow[N / x](\lambda y \cdot P) \equiv \lambda y . P$
$(f)(x \in \mathrm{FV}(P) \wedge y \notin \mathrm{FV}(N)) \rightarrow[N / x](\lambda y \cdot P) \equiv \lambda y \cdot[N / x] P$
$(g)(x \in \mathrm{FV}(P) \wedge y \in \mathrm{FV}(N)) \rightarrow[N / x](\lambda y \cdot P) \equiv \lambda z \cdot[N / x][z / y] P$

751. (a) $[x / x] M \equiv M$
(b) $x \notin \mathrm{FV}(M) \rightarrow[N / x] M \equiv M$
(c) $x \in \mathrm{FV}(M) \rightarrow \mathrm{FV}([N / x] M)=\mathrm{FV}(N) \cup(\mathrm{FV}(M)-\{x\})$
(d) $\operatorname{lgh}([y / x] M)=\operatorname{lgh}(M)$

752. Let $x, y, v$ be distinct, let no variable bound in $M$ be free in $v P Q$
(a) $v \notin \mathrm{FV}(M) \rightarrow[P / v][v / x] M \equiv[P / x] M$
(b) $v \notin \mathrm{FV}(M) \rightarrow[x / v][v / x] M \equiv M$
(c) $y \notin \mathrm{FV}(P) \rightarrow[P / x][Q / y] M \equiv[([P / x] Q) / y][P / x] M$
$(d) y \notin \mathrm{FV}(P) \wedge x \notin \mathrm{FV}(Q) \rightarrow[P / x][Q / y] M \equiv[Q / y][P / x] M$
(e) $[P / x][Q / x] M \equiv[([P / x] Q) / x] M$

\section{Lambda-terms: Change of bound variables, congruence}

753. [21]

754. $P$ contains an occurrence of $\lambda x . M$.

755. $y \notin \mathrm{FV}(M)$

756.

$$
\begin{array}{r}
(\lambda x \cdot M \equiv \lambda y \cdot[y / x] M):=\text { change of bound variable } \\
(\alpha \text {-conversion in } P)
\end{array}
$$

757. $\left(P \equiv_{\alpha} Q\right) \leftrightarrow P$ can be converted to $Q$ by a finite (or empty) number of changes (756) 
758. $\left(P \equiv_{\alpha} Q\right):=P$ is congruent to $Q:=P \alpha$-converts to $Q$

759

$$
P \equiv_{\alpha} Q \rightarrow \mathrm{FV}(P)=\mathrm{FV}(Q)
$$

760. $\equiv_{\alpha}$ is an equivalence relation.

761. Removing the condition on bounded variables in $M$, (752) also holds for $\equiv_{\alpha}$.

762.

$$
\left(M \equiv_{\alpha} M^{\prime}\right) \wedge\left(N \equiv_{\alpha} N^{\prime}\right) \rightarrow[N / x] M \equiv_{\alpha}\left[N^{\prime} / x\right] M^{\prime}
$$

763. (762) shows that substitution is well-behaved regarding $\equiv_{\alpha}$.

764. We can think of $\equiv$ and $\equiv_{\alpha}$ as being identical.

\section{Lambda-terms: Simultaneous substitution}

765. [21]

766. See (750).

767.

$\left[N_{1} / x_{1}, \ldots, N_{n} / x_{n}\right] M:=$ simultaneous substitution for $n \geq 2$

768. $\left[N_{1} / x_{1}, \ldots, N_{n} / x_{n}\right] M$ can be different from $\left[N_{1} / x_{1}\right] \ldots\left[N_{n} / x_{n}\right] M$.

\section{Lambda-terms: $\beta$-reduction}

769. [21]

770.

$$
(\lambda x \cdot M) N:=\beta \text {-redex of }[N / x] M
$$


771.

$$
[N / x] M:=\text { contractum of }(\lambda x . M) N
$$

772. In this context $\supseteq$ means contains an occurrence of a $\lambda$-term.

773.

$$
\left.(P \supseteq(\lambda x . M) N) \wedge P^{\prime} \equiv[[N / x] M] P /(\lambda x . M) N\right) \quad \leftrightarrow \quad P \triangleright_{1 \beta} P^{\prime}
$$

774. $\left(P \triangleright_{1 \beta} P^{\prime}\right):=P \beta$-contracts to $P^{\prime}$ (contraction of the redex-occurrence in $P$ )

775. $\left(P \triangleright_{\beta} P^{\prime}\right):=P \beta$-reduces to $Q$

iff $P$ can be changed to $Q$ by a finite number of $\beta$-contractions and changes of bound variables

776. $\beta$-reduction not necessarily simplifies a term; it terminates when there are no redexes.

\section{Lambda-terms: $\beta$-normal form}

777. [21]

778. $\beta$-normal form $(\beta$-nf $):=$ a term with no $\beta$-redexes

779. $\beta$-nf (or $\lambda \beta$-nf) $:=$ class of all $\beta$-normal forms

780. $P \triangleright_{1 \beta}(Q$ in $\beta$-nf $) \rightarrow Q:=\beta$-normal form of $P$

781. $P, Q:=$ terms

782. A term can have a normal form and also an infinite reduction.

783. $\Omega \equiv(\lambda x . x x)(\lambda x . x x)$

784. $\Omega$ is not a normal form (it always reduces to itself)

785. $\Omega:=$ minimal (it cannot be reduced to any different term) 
786. The $\alpha$-steps (756) are allowed in $\beta$-reductions in order to change bound variables at the beginning of the reduction and therefore avoid having to change variables while substituting.

787. lambda-calculus $\sim$ programming language $\sim \succ$ two $\beta$-reductions reach the same normal form $\sim \succ$ the end-result is independent of the path $\sim \succ$ Church-Rosser theorem: the normal form of a term is unique

788. $\triangleright_{\beta}, \mathrm{FV}$, and $\supseteq$ : (nothing new can be introduced during a reduction)

$$
P \triangleright_{\beta} Q \rightarrow \mathrm{FV}(P) \supseteq \mathrm{FV}(Q)
$$

789. Substitution and $\triangleright_{\beta}$ : $\left(\triangleright_{\beta}\right.$ is preserved by substitution)

$$
\left(P \triangleright_{\beta} P^{\prime}\right) \wedge\left(Q \triangleright_{\beta} Q^{\prime}\right) \rightarrow[P / x] Q \triangleright_{\beta}\left[P^{\prime} / x\right] Q^{\prime}
$$

790. Church-Rosser theorem for $\triangleright_{\beta}$

$$
\left(P \triangleright_{\beta} M\right) \wedge\left(P \triangleright_{\beta} N\right) \rightarrow \exists T: M\left(\triangleright_{\beta} T\right) \wedge\left(N \triangleright_{\beta} T\right)
$$

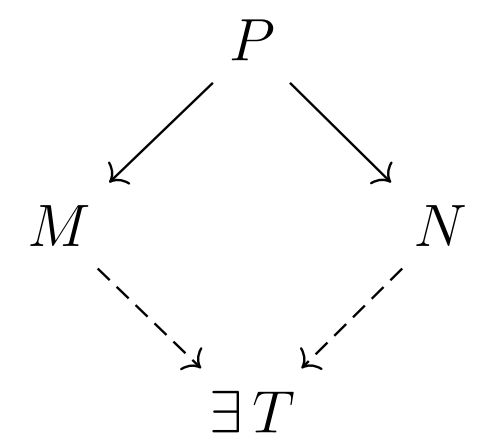

791. The property in (790) is called confluence.

792. The theorem (790) states that $\beta$-reduction is confluent.

793. If $P$ has a $\beta$-normal form, it is unique modulo $\equiv_{\alpha}$

$$
\left(P \triangleright_{\beta} M\right) \wedge\left(P \triangleright_{\beta} N\right) \rightarrow M \equiv_{\alpha} N
$$

794. $\beta$-nf is the smallest class such that:

$$
\text { (a) } \forall a(a \in \beta \text {-nf })
$$



(b) $M_{1}, \ldots, M_{n} \in \beta$-nf $\rightarrow \forall a: a M_{1} \ldots M_{n} \in \beta$-nf
(c) $M \in \beta$-nf $\rightarrow \lambda x . M \in \beta$-nf

795. $a:=$ atoms

796.

$$
\begin{aligned}
& \left(M \equiv a M_{1} \ldots M_{n}\right) \wedge\left(M \triangleright_{\beta} N\right) \wedge\left(M_{i} \triangleright_{\beta} N_{i} \text { for } i=1, \ldots n\right) \rightarrow \\
& \quad \rightarrow N \equiv a N_{1} \ldots N_{n}
\end{aligned}
$$

\section{Lambda-terms: $\beta$-equality}

797. [21]

798.

$$
\begin{array}{r}
P={ }_{\beta} Q \leftrightarrow \quad \exists P_{0}, \ldots, P_{n}(n \geq 0): \\
(\forall i \leq n-1)\left(P_{i} \triangleright_{1 \beta} P_{i+1} \vee P_{i+1} \triangleright_{1 \beta} P_{i} \vee P_{i} \equiv_{\alpha} P_{i+1}\right), \\
P_{0} \equiv P, \quad P_{n} \equiv Q
\end{array}
$$

799. $\left(P={ }_{\beta} Q\right):=P$ is $\beta$-equal $(\beta$-convertible $)$

800. $\left(P={ }_{\beta} Q\right)$ means $Q$ can be obtained from $P$ by a finite (or empty) (reversed) $\beta$-contractions and changes of variables.

801.

$$
\left(P={ }_{\beta} Q\right) \wedge\left(P \equiv_{\alpha} P^{\prime}\right) \wedge\left(Q \equiv{ }_{\alpha} Q^{\prime}\right) \rightarrow P^{\prime}={ }_{\beta} Q^{\prime}
$$

802. Substitution lemma for $\beta$-equality

$$
\left(M={ }_{\beta} M^{\prime}\right) \wedge\left(N={ }_{\beta} N^{\prime}\right) \rightarrow[N / x] M={ }_{\beta}\left[N^{\prime} / x\right] M^{\prime}
$$

803. Church-Rosser theorem for $=_{\beta}$

$$
P={ }_{\beta} Q \rightarrow \exists T:\left(M \triangleright_{\beta} T\right) \wedge\left(N \triangleright_{\beta} T\right)
$$




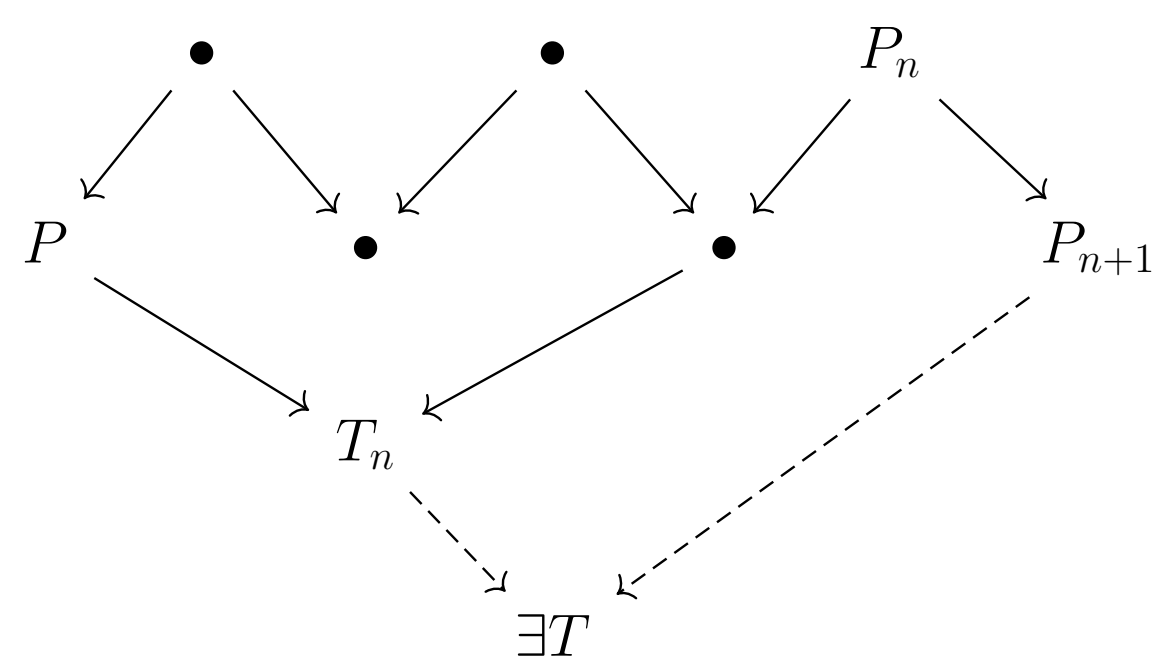

Two $\beta$-convertible terms can both be reduced to the same term.

804. $\beta$-convertibility is called $=$.

805.

$$
\left(P={ }_{\beta} Q\right) \wedge(Q:=\beta \text {-normal form }) \rightarrow P \triangleright_{\beta} Q
$$

806.

$$
\left(P={ }_{\beta} Q\right) \rightarrow(P, Q:=\text { same } \beta \text {-nf }) \underline{\vee}(P, Q:=\text { no } \beta \text {-nf })
$$

807.

$$
(P, Q \in \beta \text {-nf }) \wedge\left(P={ }_{\beta} Q\right) \rightarrow P \equiv_{\alpha} Q
$$

808. the relation $\beta$-nf is non-trivial $\sim \succ$ not all terms are $\beta$-convertible to each other

809. e.g., since $\lambda x y . x y \not_{\alpha} \lambda x y . y x$ then $\lambda x y . x y \neq_{\beta} \lambda x y . y x$

810. Uniqueness of normal form: A term is $\beta$-equal to at most one $\beta$ normal form, modulo changes of bound variables.

811.

$$
\begin{aligned}
& (a, b:=\text { atoms }) \wedge\left(a M_{1} \ldots M_{m}={ }_{\beta} b N_{1} \ldots N_{n}\right) \rightarrow \\
& \quad \rightarrow(a \equiv b) \wedge(m=n) \wedge\left(M_{i}={ }_{\beta} N_{i}, \forall i \leq m\right)
\end{aligned}
$$

812. terms without normal forms $\sim \succ$ computed for ever (without reaching a result) 
813. $\lambda$ I-terms

(a) $v_{i}, c_{i}:=\lambda \mathrm{I}$-terms (atoms)

(b) $(M, N:=\lambda$ I-terms $) \rightarrow((M N):=\lambda$-term (application) $)$

(c) $(M:=\lambda \mathrm{I}$-term $\wedge x:=$ free variable in $M) \rightarrow$ $\rightarrow((\lambda x . M):=\lambda \mathrm{I}$-term (abstraction) $)$

814. $(\lambda I$-term $:=$ has a normal form $) \rightarrow$ (all its subterms have a normal form)

\section{Simple typing, Church-style}

815. [21]

816. mathematics $\sim \succ$ definition + function $\sim \succ$ statement of the kind (inputs + outputs)

817. $\lambda$-calculus $\sim \succ$ modify $\lambda \sim \succ$ attach expressions to terms (called types) $\sim \succ$ like labels (to denote input/output sets)

818. two approaches

(i) Church-style (explicit or rigid)

(ii) Curry-style (implicit)

819. Church-style $\sim \succ$ term's type is a built-in part of the term

820. atomic types $:=$ finite/infinite sequence of symbols

821. Simple types

(a) $(\forall a: a:=$ atomic type $) \rightarrow(a:=$ type $)$

(b) $(\sigma, \tau:=$ types $) \rightarrow((\sigma \rightarrow \tau):=$ function type $)$

822. atomic type $\sim \succ$ denotes a set 
823. $\mathrm{N}:=$ atomic type for the set of natural numbers

824. $(\sigma \rightarrow \tau):=$ set of functions from $\sigma$ (domain) to $\tau$ (range)

825. $(\mathrm{N} \rightarrow(\mathrm{N} \rightarrow \mathrm{N})):=$ set of functions from numbers to functions

826. $(\rho \rightarrow \sigma \rightarrow \tau) \equiv(\rho \rightarrow(\sigma \rightarrow \tau))$

(association from right to left)

\section{Typed $\lambda$-calculus}

827. [21]

828. $x:=$ untyped variable

829. $\tau, \sigma:=$ types

830. $\exists_{\infty}:=$ there is an infinite number

831. Typed variables

$$
x^{\tau}:=\text { variable of type } \tau
$$

(a) (consistency condition) $\exists x:\left(\exists x^{\tau} \exists x^{\sigma}\right) \wedge(\tau \not \equiv \sigma)$

(b) $\forall \tau \exists_{\infty} x_{i}^{\tau}$

832. $x^{\tau} \in \tau$

833. $x^{\mathrm{N}}:=$ arbitrary number

834. $x^{\mathrm{N} \rightarrow \mathrm{N}}:=$ function

835. $x^{\tau}:=$ typed variables

836. $c^{\tau}:=$ typed atomic constants

837. Simply typed $\lambda$-terms

$$
\text { (a) } x^{\tau}, c^{\tau}:=\text { typed } \lambda \text {-terms }
$$


(b) $\left(M^{\sigma \rightarrow \tau}, N^{\sigma}:=\right.$ typed $\lambda$-terms $) \rightarrow\left(M^{\sigma \rightarrow \tau} N^{\sigma}\right)^{\tau}:=$ typed $\lambda$-term of type $\tau$

(c) $\left(x^{\sigma}:=\right.$ typed variable $) \wedge\left(M^{\tau}:=\right.$ typed $\lambda$-term $) \Rightarrow$

$\Rightarrow\left(\lambda x^{\sigma} \cdot M^{\tau}\right)^{\sigma \rightarrow \tau}:=$ typed $\lambda$-term of type $\sigma \rightarrow \tau$

838. $M^{\tau}:=$ typed term

839. $M^{\tau} \in \tau$

840. $\left(M^{\sigma \rightarrow \tau}:=\right.$ function $\phi$ from $\sigma$ to $\left.\tau\right) \wedge\left(N^{\sigma}:=\right.$ member $a$ of $\left.\sigma\right) \Rightarrow$ $\Rightarrow\left(M^{\sigma \rightarrow \tau} N^{\sigma}\right)^{\tau}:=\phi(a) \in \tau$

841. e.g., $\quad \overline{0}^{\mathrm{N}}($ atom $):=$ zero; $\quad \bar{\sigma}^{\mathrm{N} \rightarrow \mathrm{N}}:=$ successor function

\section{The Sequent Calculus LJ}

842. [22]

843. LJ := intuitionistic logic

844. The following notation is an abbreviation for an inductive definition

$$
A::=X \mid A \rightarrow A \text {. }
$$

845. $::=$ is a definition by induction.

846. Note that in (844), at the same time that the inductive definition is given, it is also said that the propositional variable $\mathrm{X}$ and the formula A will be used to denote elements of the set being defined.

847. (844) := grammar for a version of LJ (the implication is the sole connective)

848. $X \in \mathcal{V}_{\mathcal{F}}:=$ infinite set of propositional variable names

849. $A, B, C:=$ formulas 
850. named formula := pair (formula, name)

851. $\Gamma:=$ set of named formulas

852. $(\Gamma \vdash A):=$ sequent of LJ

853. (A, A's name $) \notin \Gamma \rightarrow((\Gamma, A) \equiv(\Gamma \cup\{A\}))$

854. Irrelevant formulas in axioms are admitted.

855. Rules of LJ:

$$
\begin{gathered}
\frac{\Gamma, A, A \vdash B}{\Gamma, A \vdash A}{ }^{A x} \text { Cont } \\
\frac{\Gamma \vdash A \quad \Gamma, B \vdash C}{\Gamma, A \rightarrow B \vdash C} I_{L} \\
\frac{\Gamma \vdash A \quad \Gamma, A \vdash B}{\Gamma \vdash A \rightarrow B} I_{R} \\
\Gamma \vdash B
\end{gathered}
$$

\section{The Sequent Calculus LJT}

856. $[22,23]$

857. $(\Gamma ; \vdash A),(\Gamma ; A \vdash A):=$ sequents of LJT

858. $\Gamma:=$ set of named formulas

859. stoup $:=$ the special place between $;$ and $\vdash$

860. $\exists_{\leq 1}$ formula in the stoup.

861. Rules of LJT:

$$
\begin{array}{cc}
\frac{\Gamma, A ; A \vdash B}{\Gamma ; A \vdash A} A x & \frac{\Gamma, A ; \vdash B}{\Gamma o n t} \\
\frac{\Gamma ; \vdash A \quad \Gamma ; B \vdash C}{\Gamma ; A \rightarrow B \vdash C} I_{L} & \frac{\Gamma, A ; \vdash B}{\Gamma ; \vdash A \rightarrow B} I_{R}
\end{array}
$$


862. Head-cut rule: (in the stoup)

$$
\frac{\Gamma ; \Pi \vdash A \quad \Gamma ; A \vdash B}{\Gamma ; \Pi \vdash B} C_{H}
$$

863. Mid-cut rule: (not in the stoup)

$$
\frac{\Gamma ; \vdash A \quad \Gamma, A ; \Pi \vdash B}{\Gamma ; \Pi \vdash B} C_{M}
$$

864. $X:=$ formula

865. $(\Pi=\emptyset) \vee(\exists ! X \in \Pi)$

\section{Translation of proofs from LJ to LJT}

866. $[22,23]$

867. Irrelevant formulas in axioms are admitted.

868. In the following, $\rightsquigarrow$ means translation from LJ to LJT.

869.

$$
\overline{\Gamma, A \vdash A}^{A x} \rightsquigarrow \overline{\frac{\Gamma, A ; A \vdash A}{\Gamma, A}}^{A x} \text { Cont }
$$

870. $(A \rightarrow B) \in \Gamma$

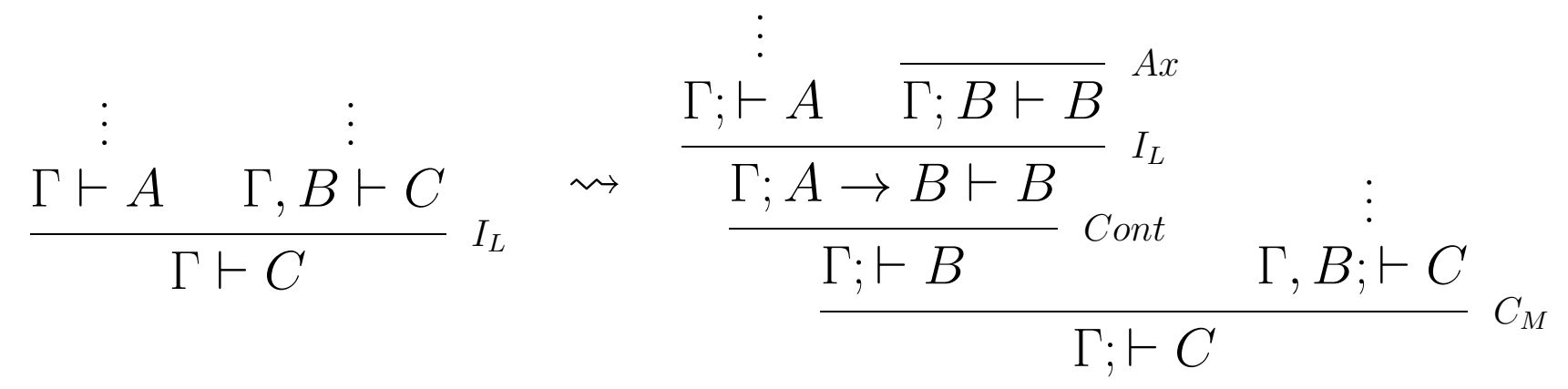

871.

$$
\frac{\Gamma, A \vdash B}{\Gamma \vdash A \rightarrow B} I_{R} \leadsto \frac{\Gamma, A ; \vdash B}{\Gamma ; \vdash A \rightarrow B} I_{R}
$$


872.

$$
\frac{\Gamma \vdash A \quad \Gamma, A \vdash B}{\Gamma \vdash B} C u t \quad \rightsquigarrow \quad \frac{\Gamma ; \vdash A \quad \Gamma, A ; \vdash B}{\Gamma ; \vdash B} C_{M}
$$




\section{Proofs in Natural Deduction}

873. $[19,20]$

874. Recall the following rules for natural deduction

(i) Hypothesis: $A$

(ii) Introductions:

$$
\begin{aligned}
& \text { [A] } \\
& \frac{A \quad B}{A \wedge B} \wedge \mathcal{I} \quad \frac{B}{A \rightarrow B} \rightarrow \mathcal{I} x \quad \frac{A}{\forall x . A} \forall \mathcal{I} \quad \frac{A[a / x]}{\exists x \cdot A} \exists \mathcal{I} \\
& \frac{A}{A \vee B} \vee 1 \mathcal{I} \quad \frac{B}{A \vee B} \vee 2 \mathcal{I} \quad \frac{\vdots}{\frac{\perp}{\neg A} \neg \mathcal{I}} \\
& {[A] \quad[B]} \\
& \vdots \quad \vdots \\
& \frac{B \quad A}{A \leftrightarrow B} \leftrightarrow \mathcal{I}
\end{aligned}
$$

(iii) Eliminations:

$$
\begin{array}{ccc}
\frac{A \wedge B}{A} \wedge 1 \mathcal{E} \quad \frac{A \wedge B}{B} \wedge 2 \mathcal{E} & \frac{A \rightarrow B \quad A}{B} \rightarrow \mathcal{E} \\
{[A]} & \\
\vdots & \frac{\forall x . A}{A[a / x]} \forall \mathcal{E} \\
\frac{\exists x \cdot A \quad B}{B} \exists \mathcal{E} &
\end{array}
$$

$$
\begin{aligned}
& {[A] \quad[B]}
\end{aligned}
$$

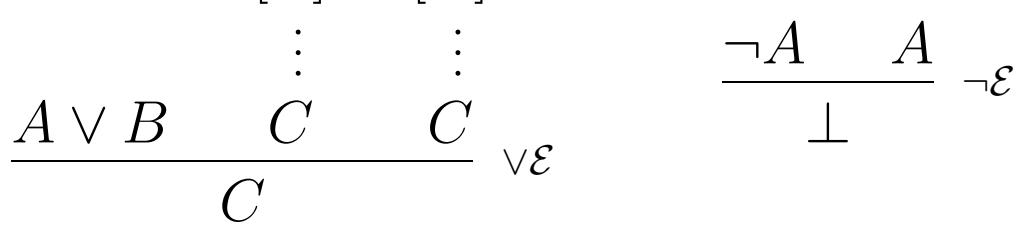




$$
\frac{A \leftrightarrow B \quad A}{B} \leftrightarrow \mathcal{E} 1 \quad \frac{A \leftrightarrow B \quad B}{A} \leftrightarrow \mathcal{E} 2
$$

(iv) Absurdity:

$$
\begin{gathered}
{[\neg A]} \\
\vdots \\
\frac{\perp}{A} \perp
\end{gathered}
$$

875. Show that $A \rightarrow(B \rightarrow C) \vdash B \rightarrow(A \rightarrow C)$.

876. Proof in natural deduction

$$
\frac{A \rightarrow(B \rightarrow C) \quad[A]_{x}}{\frac{B \rightarrow C}{\frac{C}{A \rightarrow C} \rightarrow \mathcal{I} x} \quad[B]_{y}} \rightarrow \mathcal{E}
$$

877. Proof in simply typed $\lambda$-calculus

$$
\begin{array}{rl}
z: \alpha \rightarrow(\beta \rightarrow \gamma), x: \alpha, y: \beta \vdash z: \alpha \rightarrow(\beta \rightarrow \gamma) \quad z: \alpha \rightarrow(\beta \rightarrow \gamma), x: \alpha, y: \beta \vdash x: \alpha & \\
\frac{z: \alpha \rightarrow(\beta \rightarrow \gamma), x: \alpha, y: \beta \vdash z x: \beta \rightarrow \gamma}{z} \rightarrow \mathcal{E} & z: \alpha \rightarrow(\beta \rightarrow \gamma), x: \alpha, y: \beta \vdash y: \beta \\
\frac{z: \alpha \rightarrow(\beta \rightarrow \gamma), x: \alpha, y: \beta \vdash(z x) y: \gamma}{z: \alpha \rightarrow(\beta \rightarrow \gamma), y: \beta \vdash \lambda x \cdot(z x) y: \alpha \rightarrow \gamma} & \frac{\mathcal{z} \rightarrow x}{z: \alpha \rightarrow(\beta \rightarrow \gamma) \vdash \lambda y \cdot \lambda x \cdot(z x) y: \beta \rightarrow(\alpha \rightarrow \gamma)} \rightarrow \mathcal{I} y
\end{array}
$$

878. Proof in the natural deduction with $\lambda$-terms

$$
\frac{z: A \rightarrow(B \rightarrow C) \quad[x: A]}{\frac{z x: B \rightarrow C}{\frac{(z x) y: C}{\lambda x \cdot(z x) y: A \rightarrow C} \rightarrow \mathcal{I} x}} \rightarrow \mathcal{E}
$$


879.

$$
t=\lambda y \cdot \lambda x .(z x) y=\lambda y x . z x y
$$

880. Derive Pierce's law: $((A \rightarrow B) \rightarrow A) \rightarrow A$.

881. Proof in natural deduction

882.

$$
\begin{aligned}
& \underline{[\neg A]_{v} \quad[A]_{u}} \neg \underline{\perp} \\
& \frac{\perp}{B} \perp E
\end{aligned}
$$

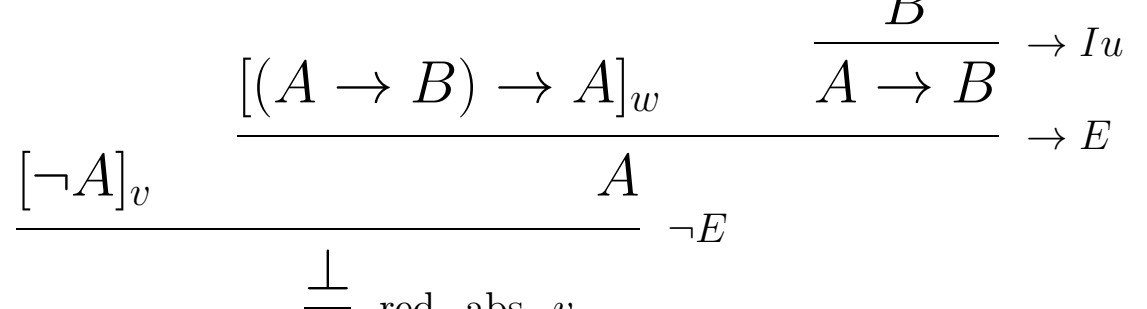

$$
\begin{aligned}
& \frac{\bar{A} \text { red. abs. v }}{((A \rightarrow B) \rightarrow A) \rightarrow A} \rightarrow I w
\end{aligned}
$$


883. Show that $\forall x(A \rightarrow B) \rightarrow(\exists x A \rightarrow \exists x B)$.

884. Proof in natural deduction

885.

$$
\begin{aligned}
& \frac{[\forall x(A \rightarrow B)]_{u}}{A \rightarrow B} \forall E \\
& {[\exists x A]_{v}} \\
& \frac{B}{\exists x B} \exists I
\end{aligned}
$$

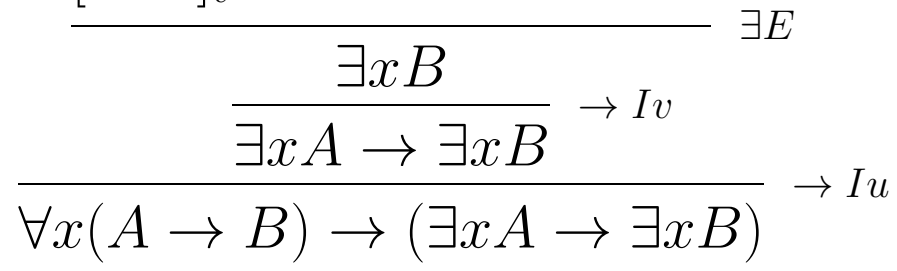

886. Show that $\vdash(A \rightarrow(B \rightarrow C)) \rightarrow(A \rightarrow B) \rightarrow(A \rightarrow C)$.

887. Proof in natural deduction

888.

$$
\begin{gathered}
\frac{[A \rightarrow(B \rightarrow C)]_{z} \quad[A]_{x} \rightarrow E}{\frac{B \rightarrow C}{\frac{C}{A \rightarrow C} \rightarrow I_{x}}} \rightarrow I_{y} \\
\frac{[A \rightarrow B]_{y} \quad[A]_{x}}{B \rightarrow B) \rightarrow(A \rightarrow C)} \rightarrow E \\
\frac{(A \rightarrow(B \rightarrow C)) \rightarrow((A \rightarrow B) \rightarrow(A \rightarrow C))}{\left(A \rightarrow I_{z}\right.}
\end{gathered}
$$




\section{Proof of sequents in LK}

889. $[3,28]$

890. Rules for the logical connectives:

891.

$$
\frac{\alpha, \Gamma \Rightarrow \Pi \quad \beta, \Gamma \Rightarrow \Pi}{\alpha \vee \beta, \Gamma \Rightarrow \Pi}(\vee \mathrm{L})
$$

892.

$$
\frac{\Gamma \Rightarrow \Lambda, \alpha}{\Gamma \Rightarrow \Lambda, \alpha \vee \beta}(\mathrm{VR} 1) \quad \frac{\Gamma \Rightarrow \Lambda, \beta}{\Gamma \Rightarrow \Lambda, \alpha \vee \beta}(\mathrm{VR} 2)
$$

893.

$$
\frac{\alpha, \Gamma \Rightarrow \Pi}{\alpha \wedge \beta, \Gamma \Rightarrow \Pi}(\wedge \mathrm{L} 1) \quad \frac{\beta, \Gamma \Rightarrow \Pi}{\alpha \wedge \beta, \Gamma \Rightarrow \Pi}(\wedge \mathrm{L} 2)
$$

894.

$$
\frac{\Gamma \Rightarrow \Lambda, \alpha \quad \Gamma \Rightarrow \Lambda, \beta}{\Gamma \Rightarrow \Lambda, \alpha \wedge \beta}(\wedge \mathrm{R})
$$

895.

$$
\frac{\Gamma \Rightarrow \Lambda, \alpha \quad \beta, \Delta \Rightarrow \Pi}{\alpha \rightarrow \beta, \Gamma, \Delta \Rightarrow \Lambda, \Pi}(\rightarrow \mathrm{L}) \quad \frac{\alpha, \Gamma \Rightarrow \Lambda, \beta}{\Gamma \Rightarrow \Lambda, \alpha \rightarrow \beta}(\rightarrow \mathrm{R})
$$

896.

$$
\frac{\Gamma \Rightarrow \Lambda, \alpha}{\neg \alpha, \Gamma \Rightarrow \Lambda}(\neg \mathrm{L}) \quad \frac{\alpha, \Gamma \Rightarrow \Lambda}{\Gamma \Rightarrow \Lambda, \neg \alpha}(\neg \mathrm{R})
$$

897. Cut rule:

$$
\frac{\Gamma \Rightarrow \Lambda, \alpha \quad \alpha, \Delta \Rightarrow \Pi}{\Gamma, \Delta \Rightarrow \Lambda, \Pi}
$$


898. Structural rules:

(i) exchange rules

$$
\frac{\Gamma, \alpha, \beta, \Delta \Rightarrow \Pi}{\Gamma, \beta, \alpha, \Delta \Rightarrow \Pi}(\mathrm{eL}) \quad \frac{\Gamma \Rightarrow \Pi, \alpha, \beta, \Lambda}{\Gamma \Rightarrow \Pi, \beta, \alpha, \Lambda}(\mathrm{eR})
$$

(ii) contraction rules

$$
\frac{\alpha, \alpha, \Gamma \Rightarrow \Pi}{\alpha, \Gamma \Rightarrow \Pi}(\text { cont } \mathrm{L}) \quad \frac{\Gamma \Rightarrow \Pi, \alpha, \alpha}{\Gamma \Rightarrow \Pi, \alpha}(\text { cont } \mathrm{R})
$$

(iii) weakening rules

$$
\frac{\Gamma \Rightarrow \Pi}{\alpha, \Gamma \Rightarrow \Pi}(w \mathrm{~L}) \quad \frac{\Gamma \Rightarrow \Pi}{\Gamma \Rightarrow \Pi, \alpha}(w \mathrm{R})
$$

899. Prove the following sequent in LK

$$
\Rightarrow A \rightarrow(B \rightarrow A) .
$$

900. Proof

$$
\begin{gathered}
\frac{A \Rightarrow A}{B, A \Rightarrow A}(w \mathrm{~L}) \\
\frac{{ }_{A \Rightarrow B \rightarrow A}}{\Rightarrow A \rightarrow(B \rightarrow A)}(\rightarrow \mathrm{R})
\end{gathered}
$$

901. Prove the following sequent in LK

$$
A \rightarrow(B \rightarrow C) \Rightarrow(A \rightarrow B) \rightarrow(A \rightarrow C) .
$$


902. Proof

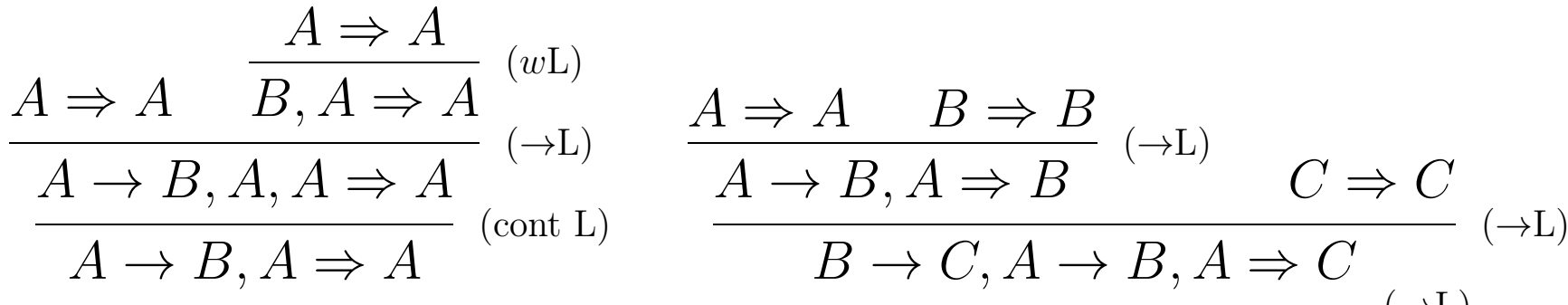

$$
\begin{aligned}
& A \rightarrow(B \rightarrow C), A \rightarrow B, A, A \rightarrow B, A \Rightarrow C \\
& A \rightarrow(B \rightarrow C), A \rightarrow B, A \rightarrow B, A, A \Rightarrow C \\
& A \rightarrow(B \rightarrow C), A \rightarrow B, A \Rightarrow C \quad \text { (cont L) } \\
& \overline{A \rightarrow(B \rightarrow C), A \rightarrow B \Rightarrow A \rightarrow C}(\rightarrow \mathrm{R}) \\
& \overline{A \rightarrow(B \rightarrow C) \Rightarrow(A \rightarrow B) \rightarrow(A \rightarrow C)}(\rightarrow \mathrm{R})
\end{aligned}
$$

903. Prove the following sequent in LK

$$
\Rightarrow A \vee \neg A \text {. }
$$

904. Proof

$$
\begin{gathered}
\frac{A \Rightarrow A}{\frac{A \Rightarrow A \vee \neg A}{(\vee \mathrm{R})}}\left(\stackrel{\mathrm{R})}{\Rightarrow A \vee \neg A, \neg A}{ }^{\frac{(\vee \mathrm{R})}{\Rightarrow A \vee \neg A, A \vee \neg A}}\right. \\
\Rightarrow \text { (cont } \mathrm{R})
\end{gathered}
$$

905. Proof

$$
\begin{aligned}
& A \Rightarrow A \\
& \Rightarrow A, \neg A(\neg \mathrm{R}) \\
& \Rightarrow A \vee \neg A, A \text { ( }(\mathrm{R}) \\
& \Rightarrow A \vee \neg A, A \vee \neg A \text { ( }(\mathrm{R}) \\
& \Rightarrow A \vee \neg A
\end{aligned}
$$


906. Prove the following sequent in LK

$$
\neg(A \wedge B) \Rightarrow \neg A \vee \neg B .
$$

907. Proof

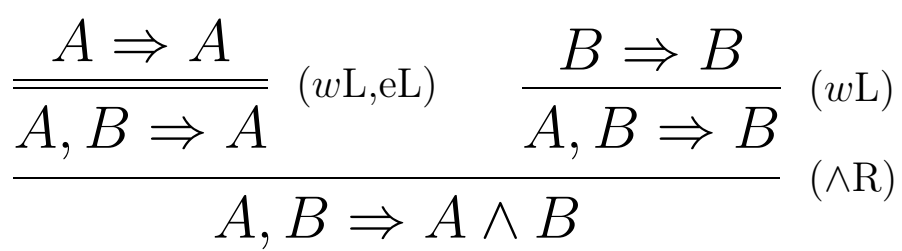

$$
\begin{aligned}
& \begin{array}{l}
\overline{\overline{\neg(A \wedge B) \Rightarrow \neg A, \neg B}}(\neg \mathrm{L}, \neg \mathrm{R}, \neg \mathrm{R}, \mathrm{e}) \\
\neg(A \wedge B) \Rightarrow \neg A \vee \neg B
\end{array}(\vee \mathrm{R}, \vee \mathrm{R}, \text { cont } \mathrm{R})
\end{aligned}
$$

908. Prove the following sequent in LK

$$
(A \rightarrow B) \rightarrow A \Rightarrow A \text {. }
$$

909. Proof

$$
\begin{aligned}
& \frac{A \Rightarrow A}{A \Rightarrow A, B}(w \mathrm{R})
\end{aligned}
$$

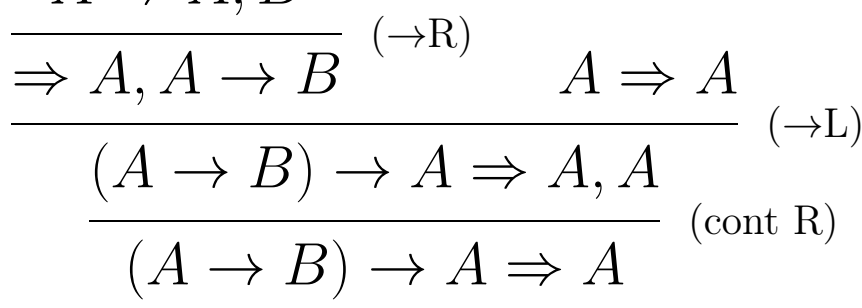

910. Prove the following sequent in LK

$$
A \rightarrow(B \rightarrow C) \Rightarrow B \rightarrow(A \rightarrow C) .
$$

911. Proof

$$
\begin{aligned}
& A \Rightarrow A \quad \frac{B \Rightarrow B \quad C \Rightarrow C}{B \rightarrow C, B \Rightarrow C}(\rightarrow \mathrm{L}) \\
& A \rightarrow(B \rightarrow C), A, B \Rightarrow C \\
& \overline{\overline{A \rightarrow(B \rightarrow C), B \Rightarrow A \rightarrow C}}(\mathrm{eL}, \rightarrow \mathrm{R}) \\
& \overline{\overline{A \rightarrow(B \rightarrow C) \Rightarrow B \rightarrow(A \rightarrow C)}}(\mathrm{eL}, \rightarrow \mathrm{R})
\end{aligned}
$$


912. Let $\mathcal{D}_{i}[S]$ be a proof tree of the sequent $S$.

913. $\mathcal{D}_{1}[A \Rightarrow B \rightarrow A]$

$$
\frac{\frac{A \Rightarrow A}{B, A \Rightarrow A} w \mathrm{~L}}{A \Rightarrow B \rightarrow A} \rightarrow \mathrm{R}
$$

914. $\mathcal{D}_{2}[A \rightarrow B, A \Rightarrow A]$

$$
\frac{{ }_{A \Rightarrow A \quad \frac{A \Rightarrow A}{B, A \Rightarrow A}}}{A \rightarrow \mathrm{L}}
$$

915. $\mathcal{D}_{3}[A \rightarrow B, A \Rightarrow B]$

$$
\frac{A \Rightarrow A \quad B \Rightarrow B}{A \rightarrow B, A \Rightarrow B} \rightarrow \mathrm{L}
$$

916. $\mathcal{D}_{4}[A \rightarrow(B \rightarrow C), A \rightarrow B, A \Rightarrow C]$

$$
\begin{array}{cc}
\vdots & \vdots \mathcal{D}_{3} \\
\frac{\mathcal{D}_{2}}{A \rightarrow B, A \Rightarrow A} & \frac{A \rightarrow B, A \Rightarrow B \quad C \Rightarrow C}{B \rightarrow C, A \rightarrow B, A \Rightarrow C}
\end{array} \rightarrow \mathrm{L}
$$

917. $\mathcal{D}_{5}[A \rightarrow(B \rightarrow C) \Rightarrow(A \rightarrow B) \rightarrow(A \rightarrow C)]$

$$
\begin{gathered}
\vdots \mathcal{D}_{4} \\
\frac{A \rightarrow(B \rightarrow C), A \rightarrow B, A \Rightarrow C}{A \rightarrow(B \rightarrow C) \Rightarrow(A \rightarrow B) \rightarrow(A \rightarrow C)} \rightarrow \mathrm{R}
\end{gathered}
$$

918. $\mathcal{D}_{6}[\Rightarrow A \vee \neg A]$

$$
\begin{aligned}
& \frac{A \Rightarrow A}{A \Rightarrow A \vee \neg A} \vee \mathrm{R} \\
& \frac{{ }_{\Rightarrow A \vee \neg A, \neg A}}{\Rightarrow \mathrm{R}} \\
& \Rightarrow A \vee \neg A
\end{aligned}
$$


919. $\mathcal{D}_{7}[A, B \Rightarrow A \wedge B]$

$$
\frac{\frac{A \Rightarrow A}{\overline{A, B \Rightarrow A} w \mathrm{~L}, \mathrm{eL}} \quad \frac{B \Rightarrow B}{A, B \Rightarrow B} w \mathrm{~L}}{A, B \Rightarrow A \wedge B} \wedge \mathrm{R}
$$

920. $\mathcal{D}_{8}[\neg(A \wedge B) \Rightarrow \neg A \vee \neg B]$

$$
\begin{gathered}
\vdots \\
\frac{\mathcal{D}_{7}}{A, B \Rightarrow A \wedge B} \\
\frac{\neg(A \wedge B) \Rightarrow \neg A, \neg B}{\overline{\neg(A \wedge B) \Rightarrow \neg A \vee \neg B}} \vee \mathrm{L}, \neg \mathrm{R}, \neg \mathrm{R}, \mathrm{eR}, \vee \mathrm{R}, \mathrm{cR}
\end{gathered}
$$

921. $\mathcal{D}_{9}[\Rightarrow A, A \rightarrow B]$

$$
\frac{\frac{A \Rightarrow A}{A \Rightarrow A, B} w \mathrm{R}}{\Rightarrow A, A \rightarrow B} \rightarrow \mathrm{R}
$$

922. $\mathcal{D}_{10}[(A \rightarrow B) \rightarrow A \Rightarrow A]$

$$
\begin{gathered}
\vdots \\
\frac{\mathcal{D}_{9}}{A \rightarrow B} \quad A \Rightarrow A \\
\hline(A \rightarrow B) \rightarrow A \Rightarrow A
\end{gathered} \rightarrow \mathrm{L}, \mathrm{cR}
$$

923. $\mathcal{D}_{11}[A \rightarrow(B \rightarrow C), A, B \Rightarrow C]$

$$
\frac{A \Rightarrow A \quad \frac{B \Rightarrow B \quad C \Rightarrow C}{B \rightarrow C, B \Rightarrow C} \rightarrow \mathrm{L}}{A \rightarrow(B \rightarrow C), A, B \Rightarrow C} \rightarrow \mathrm{L}
$$


924. $\mathcal{D}_{12}[A \rightarrow(B \rightarrow C) \Rightarrow B \rightarrow(A \rightarrow C)]$

$$
\begin{gathered}
\vdots_{11} \\
\frac{A \rightarrow(B \rightarrow C), A, B \Rightarrow C}{\overline{A \rightarrow(B \rightarrow C), B \Rightarrow A \rightarrow C}} \\
\hline \overline{A \rightarrow(B \rightarrow C) \Rightarrow \mathrm{L}, \mathrm{R}} \\
\mathrm{AL},-
\end{gathered}
$$

925. Suppose $\Rightarrow B, A$ and $A \Rightarrow B$.

$$
\begin{gathered}
\Rightarrow B, A \quad A \Rightarrow B \\
\Rightarrow B, B \\
\Rightarrow B
\end{gathered}
$$

926. A-cut is not contraction-free in LK.

$$
\frac{\Rightarrow B, A \quad A \Rightarrow B}{\Rightarrow B} \text { A-cut }
$$

927. $\mathcal{D}_{13}[\Rightarrow A \vee \neg A]:=$ contraction-free proof of LEM in LK with A-cut if $A$-cut is an atomic (primitive) rule.

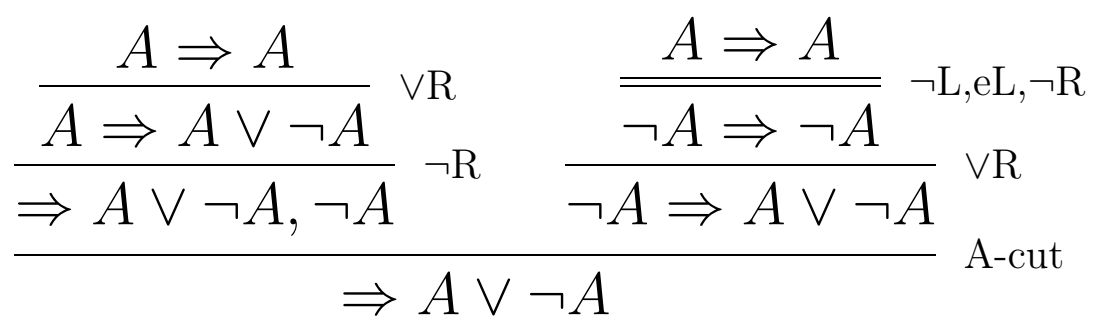

928. Suppose $\Rightarrow A, B$.

$$
\begin{gathered}
\frac{\Rightarrow A, B}{\Rightarrow A \vee B, A} \vee \mathrm{R}, \mathrm{eR} \\
\frac{A \vee B, A \vee B}{} \mathrm{\vee R} \\
\Rightarrow A \vee B
\end{gathered}
$$

929. Suppose $\Rightarrow A \vee B$.

$$
\begin{aligned}
& \Rightarrow A \vee B \quad \frac{\frac{A \Rightarrow A}{A \Rightarrow A, B} w \mathrm{R} \quad \frac{B \Rightarrow B}{B \Rightarrow A, B}}{A \vee B \Rightarrow \mathrm{R}, \mathrm{eR}} \\
& \Rightarrow A, B
\end{aligned}
$$




\section{Proof of sequents in LJ and in LJT}

930. [22]

931. Consider LJ with the sole connective $\rightarrow$.

932. Rules of LJ:

$$
\begin{gathered}
\frac{\Gamma, A, A \vdash B}{\Gamma, A \vdash A} A x \quad \text { Cont } \\
\frac{\Gamma \vdash A \quad \Gamma, B \vdash C}{\Gamma, A \rightarrow B \vdash C} I_{L} \quad \frac{\Gamma, A \vdash B}{\Gamma \vdash A \rightarrow B} I_{R} \\
\frac{\Gamma \vdash A \quad \Gamma, A \vdash B}{\Gamma \vdash B} \text { Cut }
\end{gathered}
$$

933. Prove the following sequent in LJ

$$
\vdash(A \rightarrow(B \rightarrow C)) \rightarrow((A \rightarrow B) \rightarrow(A \rightarrow C)) .
$$

934. Proof in LJ

$$
\begin{aligned}
& \frac{\frac{\overline{A \vdash A}^{A x} \quad \overline{A, B \vdash B}^{A x}}{I_{L}} \overline{A, A \rightarrow B \vdash B}}{A, A \rightarrow B, B \rightarrow C \vdash C} I_{L}{ }^{A x} I_{L} \\
& A, A \rightarrow B, A \rightarrow(B \rightarrow C) \vdash C
\end{aligned}
$$

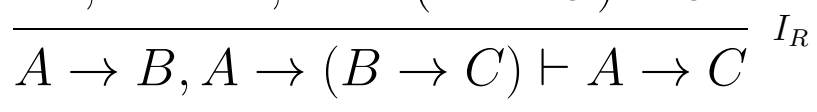

$$
\begin{aligned}
& \overline{A \rightarrow(B \rightarrow C) \vdash(A \rightarrow B) \rightarrow(A \rightarrow C)} I_{R} \\
& \overline{\vdash(A \rightarrow(B \rightarrow C)) \rightarrow((A \rightarrow B) \rightarrow(A \rightarrow C))} I_{R}
\end{aligned}
$$


935. Prove the following sequent in LJT

$$
\vdash(A \rightarrow(B \rightarrow C)) \rightarrow((A \rightarrow B) \rightarrow(A \rightarrow C)) .
$$

936. Rules of LJT (without cut):

$$
\begin{array}{cc}
\overline{\Gamma ; A \vdash A}^{A x} & \frac{\Gamma, A ; A \vdash B}{\Gamma, A ; \vdash B} \text { Cont } \\
\frac{\Gamma ; \vdash A \quad \Gamma ; B \vdash C}{\Gamma ; A \rightarrow B \vdash C} I_{L} & \frac{\Gamma, A ; \vdash B}{\Gamma ; \vdash A \rightarrow B} I_{R}
\end{array}
$$

937. Proof in LJT

$$
\begin{aligned}
& \frac{\overline{A ; \vdash A}^{D_{1}} \quad \overline{A ; B \vdash B}^{A x}}{A ; A \rightarrow B \vdash B}{ }^{I_{L}} \\
& \frac{\overline{A, A \rightarrow B ; \vdash B}}{A, A \rightarrow B ; B \rightarrow C \vdash C} \bar{A}_{I_{L}}{ }^{A x} \\
& \frac{\overline{A, A \rightarrow B ; \vdash A} D_{1}}{A, A \rightarrow B ; A \rightarrow(B \rightarrow C) \vdash C} \text { Der } I_{L} \\
& \overline{A, A \rightarrow B, A \rightarrow(B \rightarrow C) ; \vdash C}^{D e r} \\
& \overline{A \rightarrow B, A \rightarrow(B \rightarrow C) ; \vdash A \rightarrow C}^{I_{R}} \\
& \overline{A \rightarrow(B \rightarrow C) ; \vdash(A \rightarrow B) \rightarrow(A \rightarrow C)} I_{R} \\
& \overline{\vdash(A \rightarrow(B \rightarrow C)) \rightarrow((A \rightarrow B) \rightarrow(A \rightarrow C))} I_{R}
\end{aligned}
$$

938. $D_{1}:=$

$$
\overline{A ; A \vdash A}_{A ; \vdash A}^{A x}{ }^{A n t}
$$

939.

$$
\frac{\Gamma ; A \vdash B}{\Gamma, A ; \vdash B} \text { Der }
$$

940. Der $:=$

$$
\frac{\Gamma ; A \vdash B}{\Gamma, A ; A \vdash B} \text { adding irrelevant formula }
$$




\section{Open Invitation}

Review, add content, and co-author this white paper [24,25].

Join the Open Mathematics Collaboration.

Send your contribution to mplobo@uft.edu.br.

\section{Open Science}

The latex file for this white paper together with other supplementary files are available in $[26,27]$.

\section{How to cite this paper?}

https://doi.org/10.31219/osf .io/8wck9

https://zenodo.org/record/5594984

\section{Acknowledgements}

+ Center for Open Science https://cos.io

+ Open Science Framework https://osf .io

+ Zenodo

https://zenodo.org

\section{Agreement}

The author agrees with [25]. 


\section{License}

CC-By Attribution 4.0 International [2]

\section{References}

[1] Leary, Christopher C., and Lars Kristiansen. A friendly introduction to mathematical logic, 2nd edition, 2015.

https://knightscholar geneseo . edu/geneseo-authors/6/

[2] CC. Creative Commons. CC-By Attribution 4.0 International. https://creativecommons .org/licenses/by/4.0

[3] Ono, Hiroakira. Proof Theory and Algebra in Logic. Singapore: Springer, 2019.

[4] Gabbay, Dov M., and Franz Guenthner, eds. Handbook of Philosophical Logic. Vol. 1. Dordrecht: Kluwer Academic Publishers, 2001.

[5] Lobo, Matheus P. "Hilbert-style Proof Calculus for Propositional Logic in ABC Notation." OSF Preprints, 25 Nov. 2019. https://doi.org/10.31219/osf .io/jd3gp

[6] Buss, Samuel R. "An introduction to proof theory." Handbook of Proof Theory 137 (1998): 1-78. https://bit.1y/3BnmMrk

[7] Laurent, Olivier. "Focusing in orthologic." Logical Methods in Computer Science 13 (2017).

https://lmcs . episciences .org/3808/pdf

[8] Warner, Steve. Real Analysis for Beginners. GET 800, 2020.

[9] De Swart, Harrie. Philosophical and Mathematical Logic. Springer International Publishing, 2018. 
[10] Nishimura, Iwao. "On formulas of one variable in intuitionistic propositional calculus." The Journal of Symbolic Logic 25.4 (1960): 327-331. https://doi.org/10.2307/2963526

[11] Dalla Chiara, Maria Luisa, and Roberto Giuntini. "Quantum logics." Handbook of Philosophical Logic. Springer, Dordrecht, 2002. 129-228. https://arxiv.org/pdf/quant-ph/0101028.pdf

[12] WolframAlpha. https://www.wolframalpha.com

[13] Birkhoff, Garrett, and John Von Neumann. "The logic of quantum mechanics." Annals of Mathematics (1936): 823-843.

[14] Rasga, João, and Cristina Sernadas. Decidability of Logical Theories and Their Combination. Springer International Publishing, 2020.

[15] Wikipedia. "Undecidable problem."

https://en.wikipedia.org/wiki/Undecidable_problem

[16] Wikipedia. "Decision problem."

https://en.wikipedia.org/wiki/Decision_problem

[17] Wikipedia. "Word problem."

https://en.wikipedia.org/wiki/Word_problem_(mathematics)

[18] Sørensen, Morten Heine, and Pawel Urzyczyn. Lectures on the Curry-Howard Isomorphism. Elsevier, 2006.

[19] Girard, Jean-Yves, Paul Taylor, and Yves Lafont. Proofs and types. Cambridge: Cambridge university press, 1989.

[20] Wikipedia. Dedução Natural.

https://pt.wikipedia.org/wiki/Deducao_natural

[21] Hindley, J. Roger, and Jonathan P. Seldin. Lambda-calculus and Combinators, an Introduction. Cambridge: Cambridge University Press, 2008. 
[22] Herbelin, Hugo. "A $\lambda$-calculus structure isomorphic to Gentzen-style sequent calculus structure." International Workshop on Computer Science Logic. Springer, Berlin, Heidelberg, 1994.

[23] Girard, Jean-Yves. "A new constructive logic: classic logic." Mathematical structures in computer science 1.3 (1991): 255-296.

[24] Lobo, Matheus P. "Microarticles." OSF Preprints, 28 Oct. 2019. https://doi.org/10.31219/osf .io/ejrct

[25] Lobo, Matheus P. "Simple Guidelines for Authors: Open Journal of Mathematics and Physics." OSF Preprints, 15 Nov. 2019. https://doi.org/10.31219/osf .io/fk836

[26] Lobo, Matheus P. "Open Journal of Mathematics and Physics (OJMP)." OSF, 21 Apr. 2020. https://osf .io/6hzyp/files

[27] https://zenodo.org/record/5594984

[28] Indrzejczak, Andrzej. Sequents and Trees. Springer International Publishing, 2021. https://doi .org/10 .1007/978-3-030-57145-0

\section{The Open Mathematics Collaboration}

Matheus Pereira Lobo ${ }^{1,2,3}$ (lead author, mplobo@uft.edu.br)

https://orcid.org/0000-0003-4554-1372

${ }^{1}$ Federal University of Tocantins (Brazil)

${ }^{2}$ Federal University of Northern Tocantins (Brazil)

${ }^{3}$ Universidade Aberta (UAb, Portugal) 


\section{APPENDIX}

\section{Quantum Logics: Introduction}

941. $[11,12]$

942. What logical structures one may hope to find in physical theories which, like quantum mechanics, do not conform to classical logic? [13]

943. Phase-space is a mathematical concept present in both classical and quantum theories.

944. $\mathcal{S}:=$ physical system

945. $\Sigma:=$ phase-space

946. a point in $\Sigma:=$ the "state" of $\mathcal{S}$ (ascertainable by "maximal" observations)

947. pure states $:=$ maximal pieces of information about $\mathcal{S}$ (cannot be consistently extended to a richer knowledge)

948. mixtures $:=$ non maximal pieces of information

949. $P:=$ experimental proposition about $\mathcal{S}$

950. $X:=$ all the pure states for which $P$ holds

951. $X \subseteq \Sigma$

952. events (physical qualities) $:=$ subsets of $\Sigma$

953. $X:=$ event

954. $\mathcal{P}:=$ set of all experimental propositions

955. $\mathcal{E}:=$ set of all events

956. The correspondence between $\mathcal{P}$ and $\mathcal{E}$ is many-to-one. 
957. $p:=$ pure state

958.

$(\mathcal{S}$ in state $p$ verifies both $X$ and $P) \equiv(p \in X)$

959. What is the structure of all events?

960. The power-set of any set is a Boolean algebra.

961.

$$
\mathcal{B}=\langle\mathcal{F}(\Sigma), \subseteq, \cap, \cup,-, \mathbf{1}, \mathbf{0}\rangle
$$

962. $\mathcal{B}:=$ Boolean algebra

963. $\mathcal{F}(\Sigma):=$ set of all measurable events

964. $\subseteq:=$ set-theoretic inclusion relation

965. $\cap:=$ intersection of sets ("and")

966. $\cup:=$ union of sets ("or")

967. - := relative complement of a set ("not")

968. $\mathbf{1}:=\Sigma($ total space $)$

969. $\mathbf{0}:=\emptyset$ (empty space)

970. Classical semantic behaviour:

(i) ( $p$ verifies $X \cap Y) \leftrightarrow(p \in X \cap Y) \leftrightarrow$ ( $p$ verifies both members)

(ii) ( $p$ verifies $X \cup Y) \leftrightarrow(p \in X \cup Y) \leftrightarrow(p$ verifies at least one member)

(iii) $(p$ verifies $-X) \leftrightarrow(p \notin X) \leftrightarrow(p$ does not verify $X)$

971. points of $\Sigma:=$ wave-functions

972. $\Sigma \equiv$ function-space (usually the Hibert space) 
973. In classical mechanics, the excluded middle principle holds, i.e.,

$$
p \in X \vee p \notin X .
$$

974. Quantum theory is essentially probabilistic.

975. $\psi:=$ pure state (wave function) of a quantum system

976. In a quantum system, the experimental proposition $P$, for instance, can be "the spin value in a certain direction is up".

977. We have the following cases for the assignment of probability-values:

(i) $\psi(P)=1, P$ is true,

(ii) $\psi(P)=0, P$ is false,

(iii) $\psi(P) \neq 0,1, P$ is semantically indetermined.

978. Which mathematical representative would best describe quantum experimental propositions?

979. closed subspace $:=$ closed linear subspace of Hilbert space $:=$ mathematical representative of $P$ in a quantum system

980. complete metric $:=$ metric in which every Cauchy sequence is convergent

981.

Hilbert space $(\mathcal{H}):=$ vector space over a division ring $(h \in \mathcal{H} \rightarrow h \in \mathbb{R} \vee h \in \mathbb{C} \vee h \in \mathbb{H})$ such that

(i) an inner product is defined,

(ii) $\mathcal{H}$ is metrically complete.

982. $\mathbb{H}:=$ set of quaternion numbers

983.

$$
(\mathcal{H}:=\text { separable }) \leftrightarrow(\mathcal{H} \text { admits a countable basis })
$$


984. Hereafter, let

$$
\mathcal{H}:=\text { separable Hilbert space }
$$

such that its unitary vectors correspond to wave functions of a quantum system.

985. closed subspaces of $\mathcal{H}:=$ subsets of $\mathcal{H}$ (closed under linear combinations and Cauchy sequences)

986. (985) contains the mathematical representatives of experimental propositions that are closed under finite and infinite linear combinations.

987. quantum events := mathematical representatives of experimental propositions of a quantum system

988. quantum mechanics $\sim \succ$ linear combinations of $p \sim \succ$ new pure states

989. $C(\mathcal{H}):=$ set of all quantum events

990. negation of a quantum event $:=$ orthogonal complement of the event

991. orthogonal complement of a subspace $\mathrm{V}$ of the vector space := set of vectors orthogonal to all elements of $\mathrm{V}$

992. $X, X^{\prime}, Y:=$ quantum events (closed subspaces)

993. $X^{\prime}:=$ orthogonal complement of $X$

994. $X, X^{\prime}, Y \subseteq \mathcal{H}$

995. $\psi \in X^{\prime} \leftrightarrow \psi \perp X \leftrightarrow \forall \phi \in X:(\psi, \phi)=0$

996. $(\psi, \phi):=$ inner product of $\psi$ and $\phi$

997. orthocomplement := orthogonal complement

998.

$$
\forall X \forall \psi \text { (pure states) }: \psi(X)=1 \leftrightarrow \psi\left(X^{\prime}\right)=0
$$


999.

$$
\forall X \forall \psi(\text { pure states }): \psi(X)=0 \leftrightarrow \psi\left(X^{\prime}\right)=1
$$

1000.

$$
\psi \text { verifies } X \cap Y \leftrightarrow \psi \text { verifies both members }
$$

1001. union of two closed subspaces $\not \equiv$ closed subspace

1002. supremum $\sim \succ$ connective $\boldsymbol{o r}$

1003. $X \sqcup Y:=$ supremum of $X$ and $Y$ (the smallest closed subspace including both closed subspaces $X$ and $Y$ )

1004. $X \cup Y \subset X \sqcup Y$

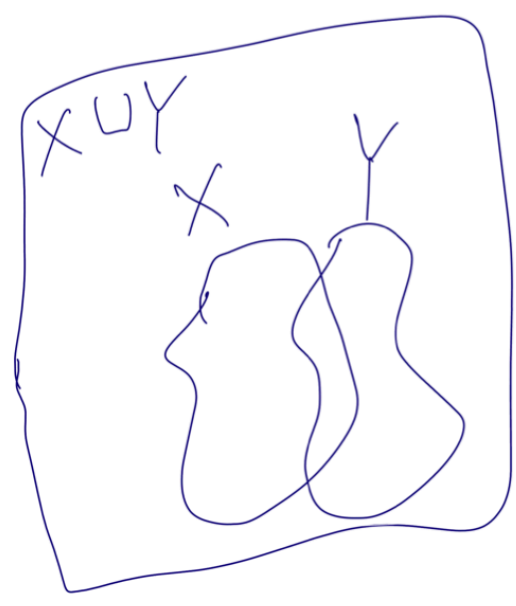

1005.

$$
\mathcal{C}(\mathcal{H})=\left\langle C(\mathcal{H}), \sqsubseteq, \sqcap, \sqcup,^{\prime}, \mathbf{1}, \mathbf{0}\right\rangle
$$

1006. $\sqsubseteq, \sqcap:=$ set-theoretic inclusion and intersection

1007. $\sqcup:=$ supremum

1008. ' $:=$ orthogonal complement

1009. $\mathbf{1}:=\mathcal{H}$ (total space)

1010. $\mathbf{0}:=$ null subspace [the singleton of the null vector (smallest subspace)] 
1011. projections $:=$ idempotent and self-adjoint linear operators

1012. $\mathfrak{P}(\mathcal{H}):=$ set of all projections $P$ of $\mathcal{H}$

1013. $\cong:=$ isomorphism

1014. $\mathfrak{P}(\mathcal{H}) \cong$ closed subspaces

1015. $\mathcal{C}(\mathcal{H})$ is not a Boolean algebra, it simulates a "quasi-Boolean behaviour".

1016. $\mathcal{C}(\mathcal{H})$ is a (not distributive) orthocomplemented orthomodular lattice,

$$
X \sqcap(Y \sqcup Z) \neq(X \sqcap Y) \sqcup(X \sqcap Z) .
$$

1017. $X \sqcup Y$ may be true even if neither member is true.

1018. It is possible for a pure state $\psi$ that

$$
\psi \notin X \wedge \psi \notin Y \rightarrow \psi \in X \sqcup Y .
$$

1019. (1016) is connected with (1018) (the superposition principle).

1020. uncertainty principle $\sim \succ$ incompatible quantities $\sim \succ$ strongly undetermined (cannot be simultaneously measured)

1021. standard quantum $\operatorname{logic}:=($ complete orthomodular lattice + closed subspaces in $\mathcal{H}) \sim \succ$ particular example of an algebraic structure 